\title{
Structure-based modeling of energy transfer in photosynthesis
}

\author{
Thomas Renger - Mohamed El-Amine Madjet • \\ Marcel Schmidt am Busch • Julian Adolphs • \\ Frank Müh
}

Received: 2 March 2013/Accepted: 8 July 2013/Published online: 7 August 2013

(C) Springer Science+Business Media Dordrecht 2013

\begin{abstract}
We provide a minimal model for a structurebased simulation of excitation energy transfer in pigmentprotein complexes (PPCs). In our treatment, the PPC is assembled from its building blocks. The latter are defined such that electron exchange occurs only within, but not between these units. The variational principle is applied to investigate how the Coulomb interaction between building blocks changes the character of the electronic states of the PPC. In this way, the standard exciton Hamiltonian is obtained from first principles and a hierarchy of calculation schemes for the parameters of this Hamiltonian arises. Possible extensions of this approach are discussed concerning (i) the inclusion of dispersive site energy shifts and (ii) the inclusion of electron exchange between pigments. First results on electron exchange within the special pair of photosystem II of cyanobacteria and higher plants are presented and compared with earlier results on purple bacteria. In the last part of this mini-review, the coupling of electronic and nuclear degrees of freedom is considered. First, the standard exciton-vibrational Hamiltonian is parameterized with the help of a normal mode analysis of the PPC. Second, dynamical theories are discussed that exploit this Hamiltonian in the study of dissipative exciton motion.
\end{abstract}

Keywords Pigment-protein complex $\cdot$ Lightharvesting $\cdot$ Förster theory $\cdot$ Redfield theory .

T. Renger $(\bowtie) \cdot$ M. Schmidt am Busch · J. Adolphs · F. Müh Institut für Theoretische Physik, Johannes Kepler Universität Linz, Altenberger Str. 69, 4040 Linz, Austria

e-mail: thomas.renger@jku.at

M. E.-A. Madjet

Center for Free-Electron Laser Science, DESY, Notkestraße 85, 22607 Hamburg, Germany
Modified Redfield theory · Generalized Förster theory · Site energies - Excitonic coupling - Spectral density

$\begin{array}{ll}\text { Abbreviations } \\ \text { BChl } & \text { Bacteriochlorophyll } \\ \text { Chl } & \text { Chlorophyll } \\ \text { CDC } & \text { Charge density coupling } \\ \text { ESP } & \text { Electrostatic potential } \\ \text { FMO } & \text { Fenna-Matthews-Olson } \\ \text { LH1 } & \begin{array}{l}\text { Core light-harvesting complex of purple } \\ \text { bacteria }\end{array} \\ \text { LH2 } & \text { Peripheral light-harvesting complex of purple } \\ & \text { bacteria } \\ \text { LHCII } & \text { Light-harvesting complex of photosystem II } \\ \text { NMA } & \text { Normal mode analysis } \\ \text { PES } & \text { Potential energy surface } \\ \text { PPC } & \text { Pigment-protein complex } \\ \text { PSI } & \text { Photosystem I } \\ \text { PSII } & \text { Photosystem II } \\ \text { QC } & \text { Quantum chemical } \\ \text { RC } & \text { Reaction center } \\ \text { bRC } & \text { Reaction center of purple bacteria } \\ \text { TDC } & \text { Transition density cube } \\ \text { TDDFT } & \text { Time-dependent density functional theory } \\ \text { TrEsp } & \text { Transition charge from electrostatic potentials }\end{array}$

\section{Introduction}

There are many reasons to study light-harvesting in photosynthesis. First of all, one touches the basis of life on earth. In cyanobacteria and higher plants, two photosystems work in series to convert the energy of solar photons into storable chemical energy. The first step of this 
complicated catalysis is the splitting of water in the oxygen evolving center that is connected to the reaction center of photosystem II (PSII). The electrons extracted from the water are transferred along a chain of cofactors through the photosynthetic membrane and are ultimately shuttled to photosystem I (PSI), where they are finally stored as chemical energy of the reductant NADPH. Electron transport between PSI and PSII is coupled to proton motion into the thylakoid lumen by the Q cycle. These protons are used together with those released from the water to drive the synthesis of ATP. A comprehensive review of the different aspects of primary reactions in photosynthesis was given, e.g., by G.Renger (2008).

In order to increase the absorption cross-section of the photosynthetic reaction centers (RCs), where the photochemistry starts, light-harvesting pigment-protein complexes (PPCs) are grouped around the RCs. They absorb photons and transfer the excitation energy to the RC. Whereas all known RCs possess a very similar structure [as discussed, e.g., by Renger (2012)], a large variety of lightharvesting complexes exists. There seems to be a unique strategy to transfer electrons through a photosynthetic membrane, but different strategies exist to efficiently transfer excitation energy (excitons) to the RC (van Grondelle et al. 1994; van Grondelle and Novoderezhkin 2006; Novoderezhkin and van Grondelle 2010).

A striking example of these variations is the difference between core light-harvesting complex LH1 surrounding the type II RC in purple bacteria (bRC) and the core lightharvesting complex of PSII in cyanobacteria and higher plants (Fig. 1). Whereas the cofactors of both RCs are practically arranged in an identical way, except for a slight tilt between two central pigments [Renger and Schlodder (2010), lower part of Fig. 2], known as the special pair, the light-harvesting complexes are organized in a completely different way. In LH1 we find a ring of 32 strongly interacting bacteriochlorophyll $a$ (BChl $a$ ) pigments (Roszak et al. 2003), whereas the pigments in the CP43 and CP47 core antennae of PSII are much less symmetrically placed and exhibit a larger average nearest neighbor distance (Umena et al. 2011). This difference in the organization of the antennae raises questions about the existence of different strategies for light-harvesting and about the evolutionary pressure that has led to such a significant change. Concerning the first question, the light-harvesting apparatus of purple bacteria may be seen as an excitation energy funnel, created by the pigment-pigment interaction. The excitonic coupling between the $32 \mathrm{BChl} a$ pigments in LH1 results in exciton delocalization and a shift of the energy levels such that the low-energy exciton states are somewhat lower in energy than those of the peripheral LH2 complex. In this way, a funnel is created that guides the excitons from the periphery toward the RC. Interestingly, only one excited state of the RC is low enough in energy to accept excitation energy from the low-energy exciton states in LH1. The origin of the large redshift of this state is the exchange interaction between the two special pair pigments (Warshel and Parson 1987). Recent calculations (Madjet et al. 2009) suggest that $80 \%$ of the excitonic coupling in the special pair is due to electron exchange. The latter also leads to a coupling between exciton states and charge transfer states resulting in an additional red shift of the lowenergy state of the special pair (Fig. 2). Hence, by exchanging electrons in addition to excitons, the special pair, a BChla dimer, is able to reach the same low excitation energies as the LH1 ring, a 30-mer. Comprehensive reviews on light-harvesting and trapping in the photosynthetic unit of purple bacteria were given, e.g., by Sundström et al. (1986, 1999), Law and Cogdell (2008).

In marked contrast to the bRC, in PSII-RCs no redshifted low-energy state is found, but all states are within an energy range of two (Raszewski et al. 2005) to three

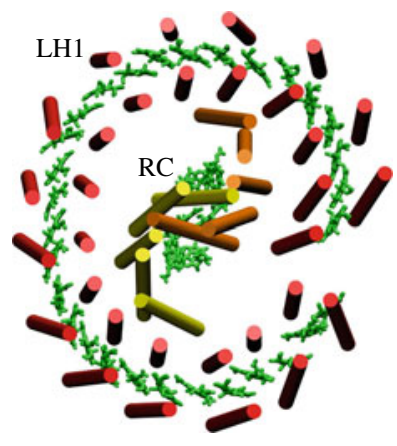

Purple Bacteria

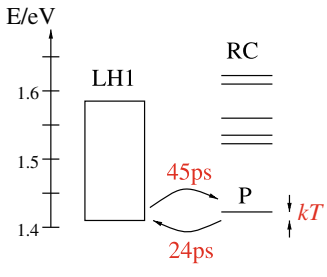

Cyanobacteria/ Higher Plants
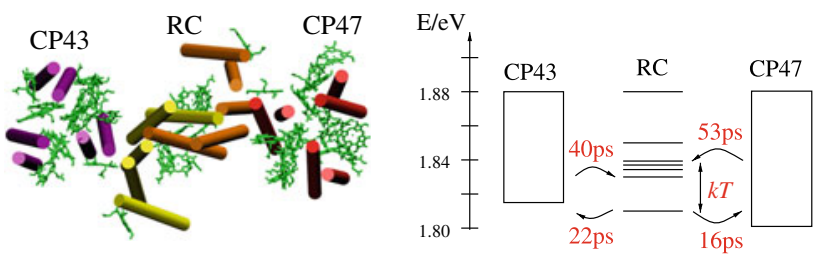

Fig. 1 Upper part Reaction center and core antenna complex LH1 of the purple bacterium Rhodopseudomonas palustris (Roszak et al. 2003). Transmembrane helices (red and yellow cylinders) are depicted together with the macrocycles of bacteriochlorophyll $a$ pigments (green). The energy levels of excited states of LH1 and $\mathrm{RC}$ and the transfer times between them, as obtained from an analysis of time-resolved spectra by Katiliene et al. (2003) are shown below the structure. $k T$ denotes the thermal energy at room temperature. Lower part Reaction center and core antenna complexes CP43 and CP47 of photosystem II of the cyanobacterium Thermosynechococcus vulcanus (Umena et al. 2011). Transmembrane helices (purple, yellow and red cylinders) are depicted together with the macrocycles of chlorophyll $a$ (and pheophytin $a$ ) pigments (green). The energy levels of excited states of CP43, RC, and CP47 and the transfer times $\mathrm{CP} 43 \leftrightarrow \mathrm{RC}$ and $\mathrm{CP} 47 \leftrightarrow \mathrm{RC}$, as calculated by Raszewski and Renger (2008), are shown below the structure. $k T$ denotes the thermal energy at room temperature 


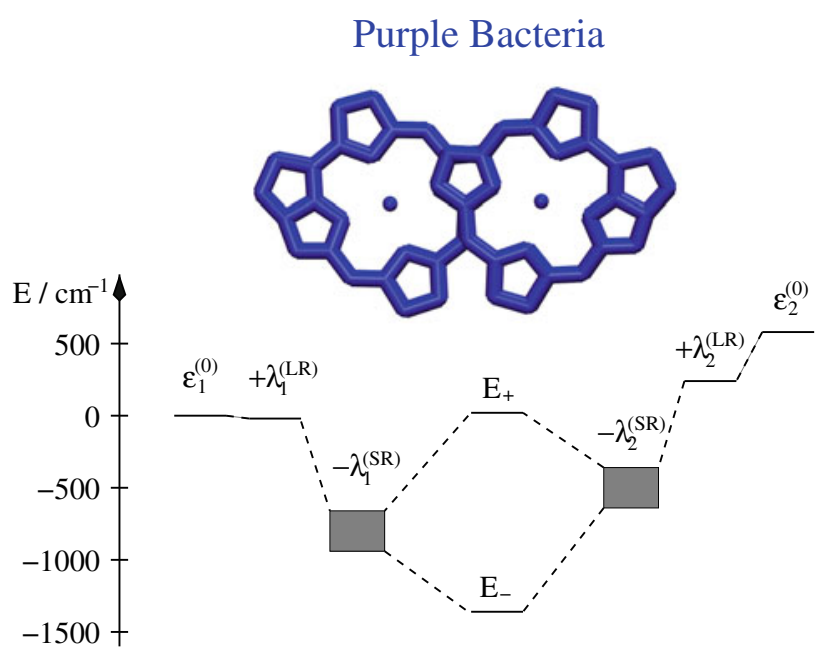

Cyanobacteria/ Higher Plants

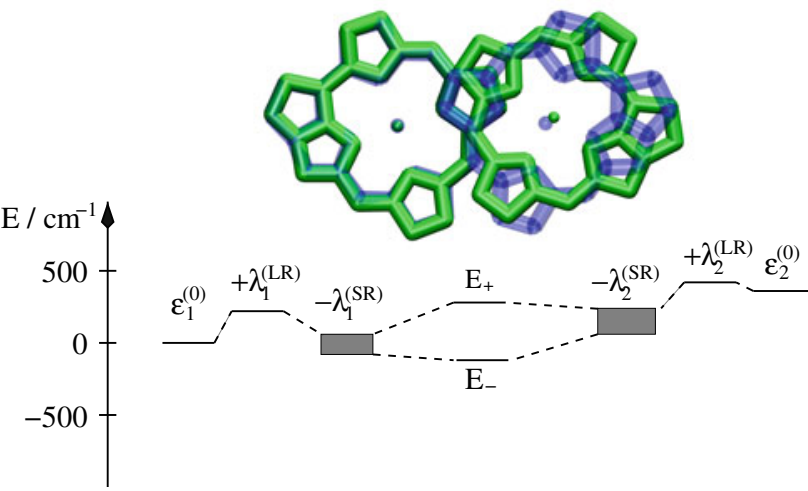

Fig. 2 Upper part Macrocycles of the two special pair bacteriochlorophylls of the reaction center from the purple bacterium $R b$. sphaeroides (Stowell et al. 1997). Below the structure, the optical transition energies $\epsilon_{i}^{(0)}$ obtained from quantum chemical calculations on the isolated monomers are related to those $\left(E_{-}\right.$and $\left.E_{+}\right)$obtained for the dimer. The $\lambda_{i}^{(\mathrm{SR})}$ and $\lambda_{i}^{(\mathrm{LR})}$ denote short- and long-range site energy shifts, respectively, obtained by using an effective two-state Hamiltonian (Madjet et al. 2009) as explained in the text.

Lower part Same as in the upper part but for the chlorophyll special pair of photosystem II of the cyanobacterium Thermosynechococcus vulcanus (Umena et al. 2011). The structure of the special pair of photosystem II (green) is overlaid by that of purple bacteria (transparent blue) in such a way that the two special pair pigments in the left half of the dimers overlap as much as possible. The details of the calculations are given in the text. The grey areas reflect uncertainties due to limitations of the quantum chemical method and the effective two-state Hamiltonian used in the analysis

(Novoderezhkin et al. 2007) times the thermal energy at room temperature (Fig. 1, bottom right part). Inspection of the two special pairs reveals a tilt in mutual orientation of the two pigments in PSII-RC with respect to bRC (Fig. 2), as noted above. Whereas there is perfect overlap of $\pi$ electron wavefunctions in the latter, in the former this overlap is weakened by the change in mutual orientation of the two special pair chlorophylls. As a consequence, the excitation energies in the special pair of photosystem II are much less influenced by short-range effects as that of purple bacteria.

The weakening of $\pi$-electron overlap in the special pair of photosystem II, which effectively switched off electron exchange, might have been an important event in evolution, since it raised the HOMO levels of the special pair pigments and thereby the oxidation potential. This likely contributes to the ability of cyanobacteria and higher plants to use water as an electron source. [A review of the optical properties of PSII-RCs, including a comparison to the bRC, is given, e.g., by Renger and Schlodder (2010)]. Although, we are not aware of a direct evidence for the above hypothesis, it is an interesting possibility that could also explain the different structure of core antennae in purple bacteria and cyanobacteria/higher plants: in cyanobacteria and higher plants, electron exchange could not be used anymore to create an excitation energy sink in the RC, since the low-energy excited states in a LH1 type antenna with chlorophyll would have been much too low in energy for efficient transfer to the RC. Therefore, the whole antenna system was restructured and a different strategy had to be developed for creating pathways for efficient transfer of excitation energy.

Another type of excitation energy funnel is realized in the rod-shaped outer antenna of cyanobacteria, the phycobilisomes. Phycobilisomes contain different forms of open tetrapyrrole pigments, the bilins, which are bound covalently to the protein (MacColl 1998). Three different types of biliproteins occur: those with high energy (phycoerythrins or phycoerythrocyanin), intermediate energy (phycocyanin), and low energy (allophycocyanin). These three types of proteins are assembled in a rod-like fashion, such that highenergy bilins are situated furthest from the core complex of photosystem II. Upon high-energy excitation the excitation energy is transferred along the rod toward the RC.

A similar type of excitation energy funnel may be created, using just a single type of pigment, by exploiting the pigment-protein interaction in such a way that the local optical transition energies get lower for those pigments that are closer to the RC. The prototype of such a site energy funnel (Louwe et al. 1997; Wendling et al. 2002; Adolphs and Renger 2006; Müh et al. 2007; Schmidt am Busch et al. 2011) is the FMO protein of green sulfur bacteria (Fenna and Matthews 1975; Tronrud et al. 2009) that will be discussed further below.

There is another important function of the protein in excitation energy transfer. The excess energy of excitons has to be dissipated when they relax toward lower energies. The protein dissipates this energy by dynamically modulating the site energies and excitonic couplings. This modulation is described by the spectral density of the 
pigment-protein coupling, which is the coupling-weighted density of vibrational states of the protein (Renger and Marcus 2002; Renger et al. 2012; Kell et al. 2013).

When modeling optical spectra and excitation energy transfer in PPCs, one faces two challenges:

(i) The calculation of site energies, excitonic couplings and spectral densities based on the crystal structure of these complexes. (ii) The development of dynamical theories that can take into account the excitonic (pigment-pigment) as well as the exciton-vibrational (pigment-protein) coupling on an equal footing.

Concerning (i) different strategies have been developed in the literature. For recent reviews from different perspectives see, e.g., Curutchet et al. (2007), König and Neugebauer (2012), Renger and Müh (2013). So far, quantum chemical (QC)/electrostatic two step approaches have obtained the best agreement with experimental data. In these approaches, one first determines the charge density of the electronic ground- and first-excited state and the transition density between the two by QC calculations on the pigments in vacuo. The resulting electrostatic potentials (ESP) of these quantities are then used to derive atomic partial charges, which are fitted such as to reproduce the ab initio ESP as close as possible. In a second step, these partial charges are applied in all-atom electrostatics calculations including the whole PPC.

Concerning (ii), the problem is to find a small parameter that can be used for perturbation theory. Both, the excitonic and the exciton-vibrational coupling are of similar strength. In this case, the standard Redfield (1957) and Förster (1948) theories, which are valid in the limit of strong and weak excitonic couplings, respectively, do not give the full answer. In recent years, numerically exact calculation schemes have been developed that are based on the hierarchical equation of motion approach (Ishizaki and Fleming 2009; Kreisbeck and Kramer 2012), density matrix renormalization/polynomial transformation (Chin et al. 2010) and path integral techniques (Nalbach et al. 2011). However, at present, these approaches are only applicable to small systems due to the enormous computational costs. Therefore, for large PPCs like those in Fig. 1, one still has to rely on approaches that are not fully non-perturbative like generalized Förster theory (Mukai et al. 1999; Jang et al. 2004) and modified Redfield theory (Zhang et al. 1998; Yang and Fleming 2002; Renger and Marcus 2003). Whereas the former can be applied to describe exciton transfer between aggregates of pigments with weak interaggregate excitonic couplings, the latter provides an improved description of exciton relaxation in aggregates of strongly coupled pigments.

Using a combination of these theories, the effective transfer times between the CP43 and CP47 core antennae and the PSII-RC, shown in the lower part of Fig. 1, were obtained (Raszewski and Renger 2008). The forward transfer from the antennae to the $\mathrm{RC}$ is slower by about a factor of two as compared to the back transfer from the RC. The larger number of pigments in the antennae provides an entropic factor in the free energy difference that determines this behavior. Experimental evidence for this concept was provided by Pawlowicz et al. (2007) using femtosecond mid-infrared spectroscopy. For open RCs (i.e., when no charge separation has occurred), the primary charge transfer occurs in less than one ps and, hence, every excitation that reaches the RC gets trapped by charge transfer. However, for closed RCs, the primary charge transfer slows down by a factor of up to 60 (Raszewski and Renger 2008) and the excitation energy has a high probability to escape from the RC into the antenna, where it can be quenched. The exact location and the molecular mechanism of the quenching is another interesting open question. By virtue of the exciton-back transfer, the entropic factor is used for photoprotection, because multiple excitations of the RC could lead to formation of triplet states, which could react with triplet oxygen to form singlet oxygen, a poison for the cell.

Interestingly, approximately the same transfer times as in PSII are found between the LH1 core antenna and the RC in purple bacteria (upper part of Fig. 1, Bergström et al. (1989); Visscher et al. (1989); Sundström et al. (1999); Katiliene et al. (2003)) despite the different organization of the antenna. A common property of both photosystems, which is responsible for this behavior, is the relatively large distance between the pigments in the $\mathrm{RC}$ and those in the antennae. In this way, an unwanted oxidation of antenna pigments by RC pigments is kinetically avoided.

In the remaining parts of this review, we will focus on structure-based microscopic models of light-harvesting in PPCs. We start by investigating how the Coulomb coupling between certain building blocks changes the electronic structure of excited states, using the variational principle. In this way, microscopic calculation schemes for the site energies and excitonic couplings are obtained. Afterwards, the dependence of these quantities on displacements of nuclei from their equilibrium positions is investigated by using a normal mode analysis (NMA), revealing the exciton-vibrational coupling Hamiltonian. Finally, in the spirit of the Born-Oppenheimer approximation, potential energy surfaces of localized and delocalized electronic states are introduced and standard theories of excitation energy transfer and extensions are discussed in this framework.

\section{The exciton Hamiltonian from a variational principle}

We divide the PPC into building blocks representing the pigments and the protein parts. The building blocks are 
defined such that electron exchange between different units can be neglected. In good approximation, every pigment is a separate building block. Concerning the protein, the division into building blocks is not so straightforward. A strict treatment would require to include an entire polypeptide chain of the protein into one building block. As we will see below, the actual building block identity of the protein is not so important. The only requirement that we will be left with, in the simple model derived here, is that we know the electrostatic potential of the electronic ground state of the protein. The latter can be well described by that of atomic partial charges as contained in standard molecular mechanics force fields.

First, we assume that the ground and excited state wavefunctions $\left|n_{a}\right\rangle=\varphi_{n_{a}}^{(a)}\left(\mathbf{r}_{1}, \ldots \mathbf{r}_{N_{a}}\right),\left(n_{a}=0,1,2,3, \ldots\right)$ of the isolated building blocks $a=1,2,3, \ldots N$ containing $N_{a}$ electrons with coordinates $\mathbf{r}_{1}, \ldots \mathbf{r}_{N_{a}}$ are known from the solution of the stationary Schrödinger equations $H_{a}\left|n_{a}\right\rangle=$ $E_{n_{a}}^{(a)}\left|n_{a}\right\rangle$. The full Hamiltonian of the PPC

$H=\sum_{a} H_{a}+\frac{1}{2} \sum_{a, b} \hat{V}_{a b}=H_{0}+\hat{V}$

contains, in addition to $H_{a}$, the intermolecular Coulomb coupling $\hat{V}_{a b}$ between the building blocks. The latter

$$
\begin{aligned}
\hat{V}_{a b}= & \sum_{i, j} \frac{e^{2}}{\left|\mathbf{r}_{i}-\mathbf{r}_{j}^{\prime}\right|}-\sum_{i, J} \frac{e^{2} Z_{J}}{\left|\mathbf{r}_{i}-\mathbf{R}_{J}^{\prime}\right|}-\sum_{I, j} \frac{e^{2} Z_{I}}{\left|\mathbf{R}_{I}-\mathbf{r}_{j}^{\prime}\right|} \\
& +\sum_{I, J} \frac{e^{2} Z_{I} Z_{J}}{\left|\mathbf{R}_{I}-\mathbf{R}_{J}^{\prime}\right|}
\end{aligned}
$$

contains the Coulomb coupling between the $i$ th electron at position $\mathbf{r}_{i}$ of building block $a$ with the $j$ th electron (at $\mathbf{r}_{j}^{\prime}$ ) and the $J$ th nuclei (charge number $Z_{J}$, position $\mathbf{R}_{J}^{\prime}$ ) of building block $b$ and the coupling between the $I$ th nuclei (charge number $Z_{I}$, position $\mathbf{R}_{I}$ ) of $a$ with the $j$ th electron and the $J$ th nuclei of $b$.

The eigenfunctions of $H_{0}=\sum_{a} H_{a}$ are product wavefunctions of the building blocks. Hence, we have

$H_{0}|\mathbf{n}\rangle=E_{\mathbf{n}}|\mathbf{n}\rangle$

where $\quad|\mathbf{n}\rangle=\left|n_{1} n_{2} n_{3} \ldots n_{N}\right\rangle=\prod_{a=1}^{N} \varphi_{n_{a}}^{(a)}\left(\mathbf{r}_{1}, \ldots \mathbf{r}_{N_{a}}\right) \quad$ and $E_{\mathrm{n}}=\sum_{a} E_{n_{a}}^{(a)}$.

In the following, we want to investigate the influence of the Coulomb coupling $\hat{V}=\frac{1}{2} \sum_{a, b} \hat{V}_{a b}$ between building blocks on the eigenstates $\left|\psi_{M}\right\rangle$ of the Hamiltonian $H$ (Eq. 1). We expand $\left|\psi_{M}\right\rangle$ in terms of the eigenfunctions of $H_{0}$

$$
\left|\psi_{M}\right\rangle=\sum_{n_{1} \ldots n_{N}} c_{n_{1} \ldots n_{N}}^{(M)}\left|n_{1} \ldots n_{N}\right\rangle=\sum_{\mathbf{n}} c_{\mathbf{n}}^{(M)}|\mathbf{n}\rangle
$$

and determine the coefficients $c_{\mathbf{n}}^{(M)}$ from a variational principle. The latter requires the expectation value
$\left\langle\psi_{M}|H| \psi_{M}\right\rangle$ to become minimal for the true wavefunction of $H$. If, in addition, we require $\left|\psi_{M}\right\rangle$ to be normalized, that is $\left\langle\psi_{M} \mid \psi_{M}\right\rangle=1=\sum_{\mathbf{k}}\left(c_{\mathbf{k}}^{(M)}\right)^{2}$, we minimize the expression $\Lambda=\left\langle\psi_{M}|H| \psi_{M}\right\rangle-\lambda_{M}\left\{\sum_{\mathbf{k}}\left(c_{\mathbf{k}}^{(M)}\right)^{2}-1\right\}$,

with the Lagrangian multipliers $\lambda_{M}$, which will become the eigenenergies of the states $\left|\psi_{M}\right\rangle$ later. Please note that we consider the stationary wavefunctions $|\mathbf{n}\rangle$ and $\left|\psi_{M}\right\rangle$ to be real quantities throughout this paper, i.e., for example, it holds that $\langle\mathbf{n}|H| \mathbf{m}\rangle=\langle\mathbf{m}|H| \mathbf{n}\rangle$ and the coefficients $c_{\mathbf{n}}^{(M)}$ are real.

As a condition for the minimum of $\Lambda$ we have $\partial / \partial c_{\mathbf{n}}^{(M)} \Lambda=0$, resulting in the following expression, using Eq. (4),

$$
\begin{gathered}
\frac{\partial}{\partial c_{\mathbf{n}}^{(M)}}\left(\sum_{\mathbf{k}, \mathbf{m}} c_{\mathbf{k}}^{(M)} c_{\mathbf{m}}^{(M)}\langle\mathbf{k}|H| \mathbf{m}\rangle-\lambda_{M}\left\{\sum_{\mathbf{k}}\left(c_{\mathbf{k}}^{(M)}\right)^{2}-1\right\}\right) \\
=2 \sum_{\mathbf{k}} c_{\mathbf{k}}^{(M)}\langle\mathbf{n}|H| \mathbf{k}\rangle-2 \lambda_{M} c_{\mathbf{n}}^{(M)}=0 .
\end{gathered}
$$

Hence, the following eigenvalue problem needs to be solved

$c_{\mathbf{n}}^{(M)}\left(\langle\mathbf{n}|H| \mathbf{n}\rangle-\lambda_{M}\right)+\sum_{\mathbf{k}}^{\mathbf{k} \neq \mathbf{n}} c_{\mathbf{k}}^{(M)}\langle\mathbf{n}|H| \mathbf{k}\rangle=0$

where the diagonal matrix elements read

$$
\begin{aligned}
\langle\mathbf{n}|H| \mathbf{n}\rangle & =\left\langle\mathbf{n}\left|\sum_{a} H_{a}+\frac{1}{2} \sum_{a, b} \hat{V}_{a b}\right| \mathbf{n}\right\rangle \\
& =\sum_{a} E_{n_{a}}^{(a)}+\frac{1}{2} \sum_{a, b} V_{n_{a} n_{b}, n_{a} n_{b}}^{(a b)}
\end{aligned}
$$

and the off-diagonal matrix elements read

$\langle\mathbf{n}|H| \mathbf{k}\rangle=\frac{1}{2} \sum_{a, b} V_{n_{a} n_{b}, k_{a} k_{b}}^{(a b)}, \quad(\mathbf{k} \neq \mathbf{n})$.

The matrix element of the interbuilding block Coulomb coupling $V_{n_{a} n_{b}, m_{a} m_{b}}^{(a b)}$ is obtained from the following integral over the electronic coordinates $\mathbf{r}_{i}$ of building block $a$ and $\mathbf{r}_{j}^{\prime}$ of $b$

$$
\begin{aligned}
V_{n_{a} n_{b}, m_{a} m_{b}}^{(a b)}= & \int d \mathbf{r}_{1} \ldots d \mathbf{r}_{N_{a}} \int d \mathbf{r}_{1}^{\prime} \ldots d \mathbf{r}_{N_{b}}^{\prime} \\
& \times \varphi_{n_{a}}^{(a)}\left(\mathbf{r}_{1}, \ldots \mathbf{r}_{N_{a}}\right) \varphi_{n_{b}}^{(b)}\left(\mathbf{r}_{1}^{\prime}, \ldots \mathbf{r}_{N_{b}}^{\prime}\right) \\
& \times \hat{V}_{a b} \varphi_{m_{a}}^{(a)}\left(\mathbf{r}_{1}, \ldots \mathbf{r}_{N_{a}}\right) \varphi_{m_{b}}^{(b)}\left(\mathbf{r}_{1}^{\prime}, \ldots \mathbf{r}_{N_{b}}^{\prime}\right)
\end{aligned}
$$

and contains the Coulomb coupling operator $\hat{V}_{a b}$ given above in Eq. (2). 
By using Pauli's principle for the exchange of electrons and renaming integration variables, the above matrix element can be simplified to (Madjet et al. 2006)

$$
\begin{aligned}
V_{n_{a} n_{b}, m_{a} m_{b}}^{(a b)}= & \int d \mathbf{r} \int d \mathbf{r}^{\prime} \frac{\rho_{m_{a}, n_{a}}^{(a)}(\mathbf{r}) \rho_{m_{b}, n_{b}}^{(b)}\left(\mathbf{r}^{\prime}\right)}{\left|\mathbf{r}-\mathbf{r}^{\prime}\right|} \\
& -\delta_{n_{b}, m_{b}} \sum_{J} \int d \mathbf{r} \frac{e Z_{J} \rho_{m_{a}, n_{a}}^{(a)}(\mathbf{r})}{\left|\mathbf{r}-\mathbf{R}_{J}^{\prime}\right|} \\
& -\delta_{n_{a}, m_{a}} \sum_{I} \int d \mathbf{r} \frac{e Z_{I} \rho_{m_{b}, n_{b}}^{(a)}\left(\mathbf{r}^{\prime}\right)}{\left|\mathbf{r}^{\prime}-\mathbf{R}_{I}\right|} \\
& +\delta_{n_{a}, m_{a}} \delta_{n_{b}, m_{b}} \sum_{I, J} \frac{e^{2} Z_{I} Z_{J}}{\left|\mathbf{R}_{I}-\mathbf{R}_{J}^{\prime}\right|},
\end{aligned}
$$

where the one-particle densities $\rho_{m_{a}, n_{a}}^{(a)}(\mathbf{r})$ and $\rho_{m_{b}, n_{b}}^{(b)}\left(\mathbf{r}^{\prime}\right)$ of the electrons in building block $a$ and $b$, respectively, were introduced according to

$\rho_{m, n}(\mathbf{r})=e \int d \mathbf{r}_{2} \ldots d \mathbf{r}_{N} \varphi_{m}\left(\mathbf{r}, \mathbf{r}_{2}, \ldots, \mathbf{r}_{N}\right) \varphi_{n}\left(\mathbf{r}, \mathbf{r}_{2}, \ldots, \mathbf{r}_{N}\right)$

which for $m=n$ is the charge density of the $m$ th molecular state and for $m \neq n$ is the transition density of the transition between the $m$ th and the $n$th electronic state.

Examining the eigenvalue problem in Eq. (7), it is seen that the off-diagonal matrix element $\langle\mathbf{n}|H| \mathbf{k}\rangle$ couples different electronic states $|\mathbf{n}\rangle$ and $|\mathbf{k}\rangle$. A considerable mixing of these states only occurs if the absolute magnitude of the difference in respective diagonal elements $\mid\langle\mathbf{n}|H| \mathbf{n}\rangle-$ $\langle\mathbf{k}|H| \mathbf{k}\rangle \mid$ is not much larger than this coupling. With this relation in mind and assuming that any Coulomb matrix element $V_{n_{a} n_{b}, m_{a} m_{b}}^{(a b)}$ is small compared to the difference between the first excited state of a building block and its ground state, $E_{1}^{(a)}-E_{0}^{(a)}$, we may well neglect any mixing of the ground state of the PPC with any excited state, by setting $\langle\mathbf{n}|H| \mathbf{0}\rangle=0$ for $\mathbf{n} \neq \mathbf{0}$. Therefore, the ground state of the aggregate reads

$\left|\psi_{0}\right\rangle=|0, \ldots, 0\rangle$

with energy

$E_{0}=\sum_{a} E_{0}^{(a)}+\frac{1}{2} \sum_{a, b} V_{00,00}^{(a b)}$.

The states next higher in energy are those, in which one pigment is in its first excited state, whereas all the remaining building blocks of the PPC are in their electronic ground state. Since the protein starts to absorb at much higher energies than the pigments, no mixing of the one-exciton states of the PPC involving an excited pigment with those involving an excited protein building block needs to be considered. Thus, we obtain the following eigenvalue problem for the one-exciton states $|m\rangle=\varphi_{1}^{(m)} \prod_{a}^{a \neq m} \varphi_{0}^{(a)}$ in which all building blocks are in their electronic ground state, except for pigment $m$, which is in its first excited state

$$
\begin{aligned}
& c_{m}^{(M)}\left\{E_{1}^{(m)}+\sum_{a}^{a \neq m}\left(E_{0}^{(a)}+V_{10,10}^{(m, a)}\right)\right. \\
& \left.+\frac{1}{2} \sum_{a, b}^{a \neq m, b \neq m} V_{00,00}^{(a, b)}-\lambda_{M}\right\}+\sum_{k}^{k \neq m} c_{k}^{(M)} V_{10,01}^{(k, m)}=0
\end{aligned}
$$

Three types of matrix elements need to be considered: the Coulomb coupling $V_{10,10}^{(m, a)}$ between the charge density of the excited state of pigment $m$ and the ground-state charge density of the remaining building blocks of the PPC (including pigments and the protein), the ground state charge density coupling $V_{00,00}^{(a, b)}$ between the remaining building blocks of the PPC, and the Coulomb coupling $V_{10,01}^{(k, m)}$ between the ground-to-excited state transition densities of the pigments. Note that $k$ in Eq. 15 runs over all pigments, whereas $a$ includes also the building blocks of the protein.

If we are interested in the transition energies between the ground state $\left|\psi_{0}\right\rangle$ and the one-exciton states $\left|\psi_{M}\right\rangle$, we may subtract from the diagonal elements of the above matrix the energy $E_{0}$ of the ground state (Eq. 14) resulting in

$c_{m}^{(M)}\left\{E_{m}-E_{M}\right\}+\sum_{k}^{(k \neq m)} c_{k}^{(M)} V_{k m}=0$,

with the site energy

$E_{m}=E_{1}^{(m)}-E_{0}^{(m)}+\sum_{a}\left(V_{10,10}^{(m, a)}-V_{00,00}^{(m, a)}\right)$

that is, the transition energy, at which pigment $m$ would absorb light, if it was not coupled excitonically to the other pigments, the transition energy

$E_{M}=\lambda_{M}$

between the ground state $\left|\psi_{0}\right\rangle$ and the one-exciton state $\left|\psi_{M}\right\rangle$, and the Coulomb coupling

$V_{k m}=V_{10,01}^{(k, m)}$

between the transition densities of the pigments. The latter type of coupling is termed excitonic coupling. Hence, the singly excited states of the PPC may be seen as eigenstates of the following exciton Hamiltonian

$H_{\mathrm{ex}}=\sum_{m} E_{m}|m\rangle\left\langle m\left|+\sum_{m, k}^{m \neq k} V_{k m}\right| m\right\rangle\langle k|$

which contains as diagonal matrix elements the site energies $E_{m}$ (Eq. 17) and in the off-diagonal the excitonic couplings $V_{k m}$ (Eq. 19). 
Moreover, our derivation of the exciton Hamiltonian provides us with a minimal model for the calculation of its parameters. As Eq. (17) suggests, the site energy $E_{m}$ of pigment $m$ is obtained from the transition energy $E_{1}^{(m)}-E_{0}^{(m)}$ of this pigment in vacuum and the difference in charge density coupling between the excited and the ground state of the pigment with the remaining building blocks of the PPC (including other pigments and the protein parts). The excitonic couplings $V_{k m}$ (Eq. 19) are obtained from the Coulomb interaction between transition densities of the pigments. In the following, we discuss methods that make use of these expressions.

\section{Calculation of site energies and excitonic couplings}

\section{Evaluation of Coulomb integrals}

In the methods discussed below, Coulomb integrals of the type in Eq. (11) need to be evaluated. An efficient calculation of these integrals is obtained by the use of atomic partial charges $q_{I}(m, n)$ of the building blocks that are determined from the fit of the electrostatic potential (ESP) of the respective molecular charge $(m=n)$ or transition $(m \neq n)$ densities (Madjet et al. 2006). The matrix ele-

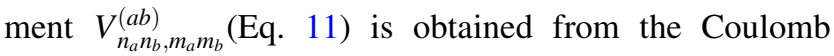
interaction between the partial charges $q_{I}^{(a)}\left(m_{a}, n_{a}\right)$ of molecule (building block) $a$ and $q_{J}^{(b)}\left(m_{b}, n_{b}\right)$ of molecule $b$ that are placed at the positions $\mathbf{R}_{I}$ of atom $I$ of $a$ and $\mathbf{R}_{J}^{\prime}$ of atom $J$ of $b$

$V_{n_{a} n_{b}, m_{a} m_{b}}^{(a b)}=\sum_{I, J} \frac{q_{I}^{(a)}\left(m_{a}, n_{a}\right) q_{J}^{(b)}\left(m_{b}, n_{b}\right)}{\left|\mathbf{R}_{I}-\mathbf{R}_{J}^{\prime}\right|}$.

In the following, these matrix elements are used to obtain site energies and excitonic couplings.

\section{Calculation of site energies}

\section{The charge density coupling (CDC) method}

In the CDC method the site energy $E_{m}$ (Eq. 17) of pigment $m$ is obtained by using Eq. (21) as (Adolphs et al. 2008)

$E_{m}=E_{0}+\frac{1}{\epsilon_{\mathrm{eff}}} \sum_{a, I, J} \frac{\left(q_{I}^{(m)}(1,1)-q_{I}^{(m)}(0,0)\right) q_{J}^{(a)}(0,0)}{\left|\mathbf{R}_{I}-\mathbf{R}_{J}^{\prime}\right|}$

where the vacuum transition energy $E_{0}=E_{1}^{(m)}-E_{0}^{(m)}$ is assumed to be the same for all chemically identical pigments and $\epsilon_{\text {eff }}$ serves to take into account screening and local field effects of the Coulomb interaction caused by the polarizability of the PPC in an effective way. In practical applications, $\epsilon_{\mathrm{eff}}$ is used also to compensate for systematic deviations in the quantum chemical calculations used to derive the partial charges. Hence, $E_{0}$ and to a certain degree $\epsilon_{\text {eff }}$ are adjustable parameters which need to be determined by comparison with experimental optical spectra. Whereas a variation of $E_{0}$ just displaces the whole spectrum along the energy axis, $\epsilon_{\text {eff }}$ has an influence on the width of the spectrum because it scales the energy differences between site energies of the pigments by a constant factor. A difficulty when applying Eq. (22) is that the charge density of those building blocks $a$, which represent titratable amino acid residues, may vary due to protonation/deprotonation reactions. Therefore, it is necessary to determine the most likely protonation state of the titratable groups, before the site energies can be calculated.

\section{Calculation of the protonation pattern of the protein}

Electrostatic methods have been developed to calculate the protonation probabilities of the titratable residues in the protein (Ullmann and Knapp 1999). In these methods, the polarizability of the protein, membrane and solvent environments are described by that of three homogeneous dielectrics with different dielectric constants. A PoissonBoltzmann equation is solved for the ESP of the titratable groups in their deprotonated and protonated states, taking into account also the ionic strength of the solvent. In addition, the potentials of the two states of suitable model compounds, representing the titratable residues in an aqueous solvent, are computed and a thermodynamic cycle is constructed revealing the electrostatic free energy difference between the protonated and deprotonated residue in the protein. To close this cycle, the experimental $\mathrm{p} K_{a}$ value of the model compound in the aqueous solvent is applied. A detailed review on the calculation of the protonation pattern of the protein in the framework of site energy calculations was given recently (Renger and Müh 2013). The calculation of protonation states in combination with CDC calculations of site energies were successfully applied to the calculation of optical spectra of PSI (Adolphs et al. 2010) and the FMO protein (Adolphs et al. 2008; Schmidt am Busch et al. 2011). The latter calculations will be discussed in more detail below.

\section{Approximations and comparison with other methods}

Besides the charge density couplings considered above, the site energies of the pigments are also influenced by dispersive and inductive interactions. In the present derivation of the exciton Hamiltonian, the inductive couplings are contained in Coulomb matrix elements of the type $V_{m_{a} 0, m_{a} k_{b}}^{(a b)}$ 
where the charge density of the ground $\left(m_{a}=0\right)$ or excited $\left(m_{a}=1\right)$ state of pigment $a$ interacts with the transition density between the ground state $|0\rangle$ and any excited state $\left|k_{b}\right\rangle$ of building block $b$. Dispersive site energy shifts arise from a correlated fluctuation of electrons of different building blocks. The respective interactions are contained in transition density couplings $V_{m_{a} m_{b}, k_{a} k_{b}}^{(a b)}$ where the transition density between the ground $\left(m_{a}=0\right)$ or excited $\left(m_{a}=1\right)$ pigment state and any other electronic state $\left|k_{a}\right\rangle$ couples with the transition density between the ground state $|0\rangle$ and any excited state $\left|k_{b}\right\rangle$ of building block $b$. These interactions, in principle, can be described by perturbation theory, as reviewed recently (Renger and Müh 2013). An application of such a perturbation theory to site energy shifts of BChla in nonpolar solvents will be discussed below.

A method that is related in spirit to CDC is the PoissonBoltzmann/Quantum Chemical method (PBQC) (Müh et al. 2007; Adolphs et al. 2008) in which the polarization of the PPC is taken into account more realistically. In $\mathrm{PBQC}$, as in the calculation of the protonation pattern of the protein discussed above, the polarization of the protein, membrane and solvent environments is described by that of three homogeneous continuous dielectrics and a PoissonBoltzmann equation is solved for the ESP of the ground and excited states of the pigments (which are represented by atomic partial charges as introduced above) and the solvation energies for these two states (resulting from inductive interactions between the charge density of the pigment and the transition density of the remaining building blocks of the PPC and Coulomb couplings between the building blocks) calculated. The latter contain the screening and local field effects that were approximated by the effective dielectric constant in the CDC method. PBQC has been successfully applied to identify energy sinks and to explain optical spectra of the FMO protein (Müh et al. 2007), the major light-harvesting complex of higher plants LHCII (Müh et al. 2010; Müh and Renger 2012), and the CP43 core antenna of PSII (Müh et al. 2012).

A critical approximation of CDC (and PBQC) is the neglect of a direct influence of the conformation of the pigments on their local transition energies, by assuming a constant $E_{0}$ value for chemically identical pigments in Eq. (22). In principle, the conformations of the pigments could be taken into account by performing QC calculations on these conformations and taking into account not only the charge density but also the QC transition energy obtained. In such an approach, one has to face the fact, that the crystallographic data are not optimized in a QC sense. QC calculations of transition energies are very sensitive to slight distortions of equilibrium geometries. It is our experience that even a constrained geometry optimization (allowing only the bond lengths and bond angles to be varied) often is not enough to obtain realistic wavefunctions of ground and excited states (as judged from difference dipole moments between excited and ground state and comparison with data from Stark spectroscopy). One way to extract the essential information from the crystal structures might be to project the conformations of the pigments on their low-frequency intramolecular normal modes (Shelnutt et al. 1998). Zucchelli et al. (2012) combined this normal mode decomposition method with a semi-empirical approach to estimate site energy shifts in the major lightharvesting complex of higher plants, LHCII. A combination of this approach with CDC or PBQC, in order to include the electrostatic pigment-protein interaction, might be worthwhile.

Other approximations of the CDC and PBQC methods concern the neglect of dispersive and inductive site energy shifts, discussed already above. Of course, these interactions are contained in a full QC treatment of the protein environment of the pigments. Besides the geometry distortion problem, such a treatment faces the problem to restrict the size of the environment that is treated by QC to keep the numerical effort manageable as well as the problem that the Schrödinger equation for a many electron wavefunction can only be solved approximately and the energies obtained might have too large uncertainties. An excellent overview of QC developments in the framework of site energy calculations of photosynthetic pigments was given recently by König and Neugebauer (2012).

Finally, we would like to point out that the CDC and PBQC approaches are two-step approaches, where the QC calculations are performed on geometry optimized pigments in vacuo and the resulting charge densities are then applied in all atom electrostatic calculations of the PPC in the second step. In this way, any influence of the protein environment on the wavefunction and the resulting charge(and transition-) densities of the pigments are neglected. One way to treat these effects is by including the external charge density by classical point charges and the external polarization by that of a homogeneous dielectric in the solution of the Schrödinger equation. Such a treatment neglects, however, Pauli repulsion between the electrons of the pigments and those of the environment. This approximation can lead to an artificial distortion of the electronic wavefunction of the pigment, an effect known as electron leakage problem (Neugebauer 2009; Schmidt am Busch and Knapp 2005; Senn and Thiel 2007). Obviously, the simplest solution of this problem is to use a two-step procedure, as described above. A quantitative evaluation of the effects neglected in such a treatment, is a rewarding task for future approaches that use a quantum mechanical description of the environment. 
Dispersive transition energy shifts of pigments in nonpolar solvents

A first step toward an inclusion of dispersive site energy shifts in PPCs was taken in a model of dispersive shifts in nonpolar solvents (Renger et al. 2008). Second-order perturbation theory in the solute-solvent interaction and a subsequent dipole and continuum approximation of the solvent were used to express the shift of the transition energy between the ground state and the $m$ th excited state of the pigments as

$\Delta E_{m 0}=\kappa_{m 0} \frac{W_{u}}{d_{u}^{2}}$

where $W_{u}$ is the solvation energy of an extended unit dipole $\left(d_{u}=1 \mathrm{D}\right)$ that is placed in a molecule-shaped cavity with dielectric constant $\epsilon=1$ (vacuum) inside, that is surrounded by a dielectric continuum with optical dielectric constant of the solvent $\epsilon=n^{2}$ outside. Here, $n$ is the refractive index of the solvent. Solvation energies of transition densities of all strong electronic transitions that start from the electronic ground state, obtained with atomic transition charges, were compared to that of an extended dipole resembling the dipole strength of the respective transition. In this way, the extent of the dipole was determined to $8.7 \AA$.

The factor $\kappa_{m 0}$ in Eq. (23) is given as

$\kappa_{m 0}=\sum_{k}^{k \neq 0, k \neq m}\left(d_{m k}^{2}-d_{0 k}^{2}\right)=\left\langle m\left|\hat{d}^{2}\right| m\right\rangle-\left\langle 0\left|\hat{d}^{2}\right| 0\right\rangle$

which is the difference in the variance of the dipole moment between the excited and the ground state. Note that the transition dipole element $d_{m 0}^{2}=d_{0 m}^{2}$ does not contribute to $\kappa_{m 0}$. From the correlation between the experimental transition energy $E_{m 0}$ and the factor $W_{u} / d_{u}^{2}$ in Eq. 23, the vacuum transition energy $E_{0}^{(m)}$ and the factor $\kappa_{m 0}$ may be obtained via a linear regression

$E_{m 0}=E_{0}^{(m)}+\kappa_{m 0} \frac{W_{u}}{d_{u}^{2}}$.

For the $0 \rightarrow 1$ transition of $\mathrm{BChl} a, E_{0}^{(1)}=13,818 \mathrm{~cm}^{-1}$ and $\kappa_{10}=117 \mathrm{D}^{2}$ were obtained by analyzing the experimental data of Limantara et al. (1997) (left part of Fig. 3). Interestingly, using instead of the molecule-shaped cavity a spherical cavity and replacing the extended unit dipole by a unit point dipole, where $W_{u} / d_{u}^{2}$ is obtained analytically (Böttcher 1973) as

$\frac{W_{u}}{d_{u}^{2}}=-\frac{1}{R^{3}} \frac{n^{2}-1}{2 n^{2}+1}$

also results in a good correlation with the same experimental data (right part of Fig. 3). From the slope $\left(\kappa_{10} / R\right)^{3}$ of the correlation plot, using the $\kappa_{10}$ from the calculation with the molecule-shaped cavity, a cavity radius of $5.8 \AA$ was inferred. Obviously, for this radius, the error compensation between using a point dipole instead of an extended dipole and a spherical instead of a moleculeshaped cavity is complete. Practically, the same vacuum transition energy $E_{0}^{(1)}=13,800 \mathrm{~cm}^{-1}$ is obtained in the simple model. We will find a similar error compensation at work in calculations of screening/local field correction factors of excitonic couplings below.

We note that the vacuum transition energy values extracted from the linear regressions in Fig. 3 are considerably larger than the $E_{0}=12,570 \mathrm{~cm}^{-1}$ inferred from CDC calculations (Schmidt am Busch et al. 2011) of site energies of BChla pigments in the FMO protein and comparison of the resulting optical spectra with experimental data (see below). A considerable part of the difference should be due to dispersive interactions, which were only implicitly taken into account in the CDC calculations by assuming them to be site-independent, as would be expected for a homogeneous dielectric. Indeed, the transition energy of BChl $a$ in $n$-dodecane, which has an optical dielectric constant $n^{2}=2$ that equals the average optical dielectric constant of PPCs (see below), amounts to $12,920 \mathrm{~cm}^{-1}$. The remaining $350 \mathrm{~cm}^{-1}$ difference to the $E_{0}$ value from CDC calculations still needs to be explained. This difference could result from other higher-order terms in the perturbation theory of the pigment-protein coupling and/or from the heterogeneity of the dispersive interaction resulting from differences in local polarizabilities of protein residues.

Finally we note that Renge and Mauring (2013), excluding those nonpolar solvents with highly polarizable atoms, and using a similar analysis as the point-dipole in a sphere model discussed above, arrived at a different vacuum transition energy of $13,340 \mathrm{~cm}^{-1}$. Hence, it might well be that the influence of dispersive interactions is smaller than it appeared from the large $E_{0}^{(1)}$ values estimated above. On the other hand, the good linear correlation found between the calculated solvation energies and experimental transition energies from Limantara et al. (1997) in Fig. 3 including the highly polarizable solvents seems to rule out artifacts by the latter.

\section{Calculation of excitonic couplings}

\section{The transition density cube (TDC) method}

The excitonic coupling between the $0 \rightarrow 1$ transitions of the pigments is obtained from Eq. 11 as

$V_{a b}=V_{01,10}^{(a b)}=\int d \mathbf{r} \int d \mathbf{r}^{\prime} \frac{\rho_{01}^{(a)}(\mathbf{r}) \rho_{10}^{(b)}\left(\mathbf{r}^{\prime}\right)}{\left|\mathbf{r}-\mathbf{r}^{\prime}\right|}$. 
Fig. 3 Left part Correlation between experimental transition energy of BChla in solvent with refractive index $n$ and the solvation energy of an extended unit dipole in a molecule-shaped cavity (upper part) with dielectric constant $\epsilon=n^{2}$ outside the cavity and $\epsilon=1$ inside. The solid line describes a linear regression of the data according to Eq. 25.

Right part Correlation between experimental transition energy in solvent with refractive index $n$ and factor $-\left(n^{2}-1\right)$ / $\left(2 n^{2}+1\right)$ resulting from the solvation energy of a point dipole in a spherical cavity (Eq. 26). The solid line describes a linear regression. The experimental data were taken from Limantara et al. (1997) and the calculations were performed in Renger et al. (2008)
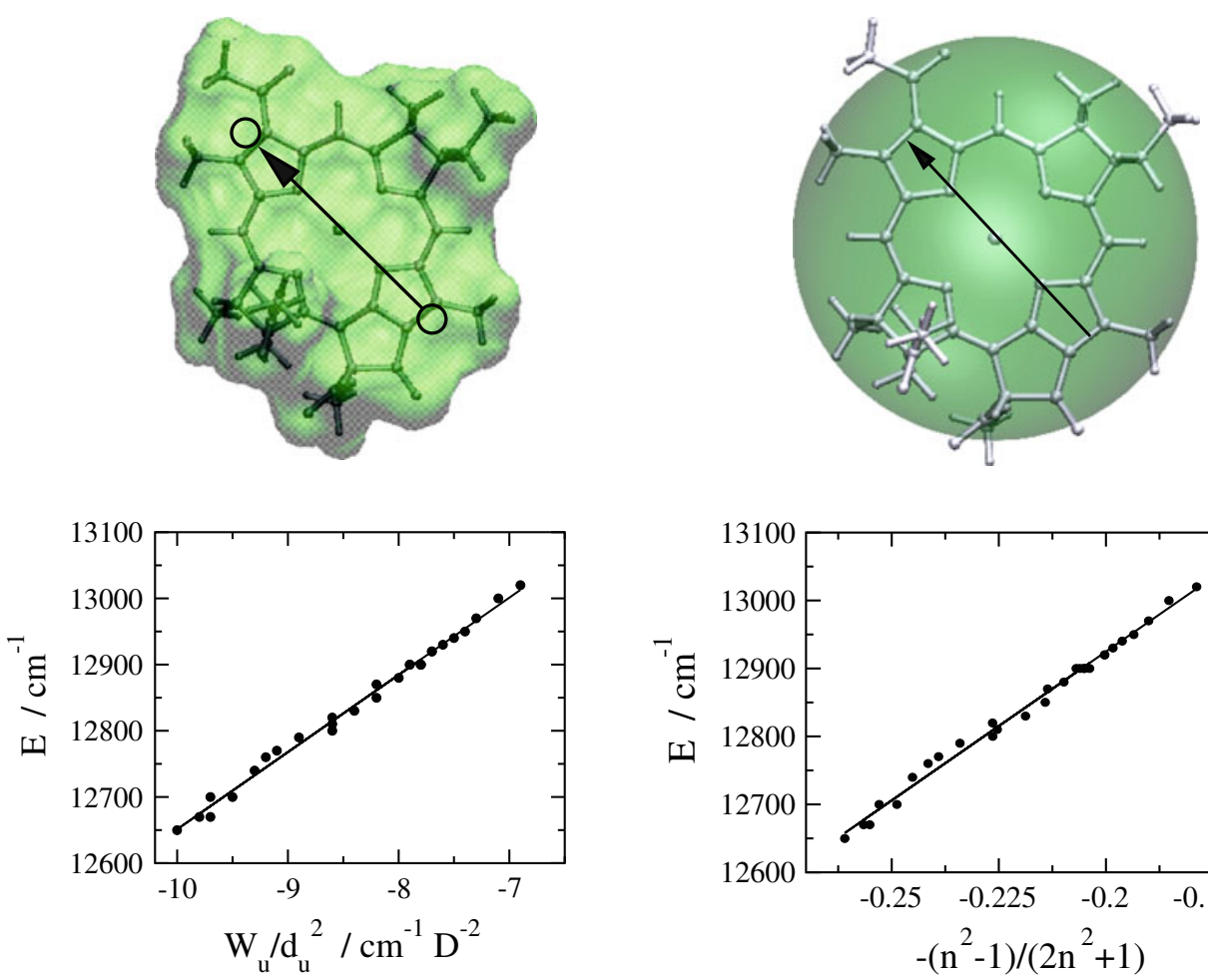

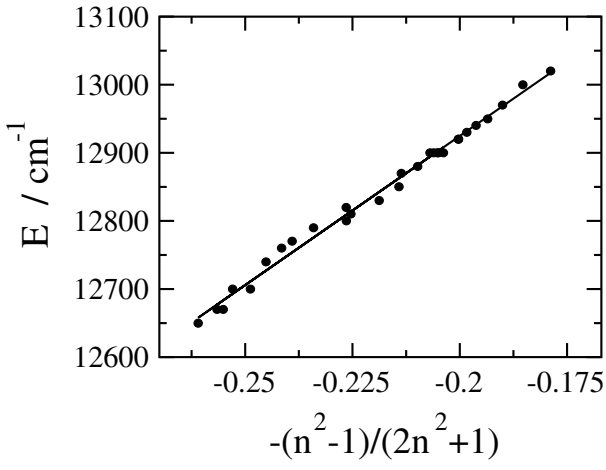

In the transition density cube method (TDC) (Krueger et al. 1998), the above 6d integral is evaluated numerically. A method of equal accuracy, but less numerical cost, is the transition charge from electrostatic potentials (TrEsp) method (Madjet et al. 2006), described next.

\section{The transition charge from electrostatic potentials (TrEsp) method}

In the TrEsp method (Madjet et al. 2006), the ESP of the transition density is fitted by that of atomic partial charges, and the excitonic coupling is obtained from Eq. (21) as

$V_{a b}=V_{01,10}^{(a b)}=f \sum_{I, J} \frac{q_{I}^{(a)}(1,0) q_{J}^{(b)}(0,1)}{\left|\mathbf{R}_{I}-\mathbf{R}_{J}^{\prime}\right|}$.

where, in addition, a scaling factor $f$ was introduced to describe screening and local field effects due to the polarizability of the protein environment in an implicit, approximate way. Two uncertainties arise when calculating excitonic couplings with TDC or TrEsp. First, different QC methods result in different transition densities (and, therefore partial charges). It seems, however, the shape of the transition densities is similar, whereas the absolute magnitude is different. Hence, there is an unknown scaling factor that needs to be determined by comparison with experimental data. The vacuum transition dipole moment of the pigments can be extrapolated from an analysis of the oscillator strength of the considered optical band of a pigment measured in different solvents. Knox and Spring (2003) performed such an analysis for chlorophyll $a(\mathrm{Chl} a), \mathrm{Chl} b, \mathrm{BChl} a$, and $\mathrm{BChl} c$. The scaling factor for the transition density and the partial charges can then be obtained from the ratio of the experimental vacuum transition dipole moment $\mathbf{d}_{10}^{\exp }$ and the transition dipole moment $\mathbf{d}_{10}$ resulting from the QC transition density or the transition charges

$\mathbf{d}_{10}=\int d \mathbf{r} \rho_{10}(\mathbf{r})=\sum_{I} q_{I}(1,0) \mathbf{R}_{I}$

Hence, scaled transition partial charges

$\tilde{q}_{I}(1,0)=\frac{\left|\mathbf{d}_{10}^{\exp }\right|}{\left|\mathbf{d}_{10}\right|} q_{I}(1,0)$

are defined, the ESP of which resembles that of the $0 \rightarrow 1$ electronic transition of the pigments in vacuo most closely.

A second uncertainty concerns the influence of the polarizability of the dielectric environment, which is described by a simple scaling factor $f$ in the TrEsp method (Eq. 28). To investigate the latter approximation and to determine $f$, the Poisson-TrEsp method was developed, which is the topic of the next subsection.

\section{The Poisson-TrEsp method}

Here, the polarizability of the PPC is described by that of a homogeneous dielectric with optical dielectric constant $\epsilon_{\mathrm{opt}}=n^{2}$, where $n$ is the refractive index. Note that, during 
the electronic transitions, involved in excitation energy transfer, the nuclei of the PPC have no time to react and, therefore, only the optical polarizability has an influence on the excitonic couplings. The mean refractive index of a PPC can be estimated (Renger et al. 2009) from comparison of the integral oscillator strength of PPC-bound and solvent-extracted pigments (Müh and Zouni 2005) as well as from microscopic simulations (Simonson and Perahia 1995). Both estimates resulted in a value $\epsilon_{\mathrm{opt}}=n^{2} \approx 2$. In order to take into account screening and local field effects by the optical polarization, molecule-shaped cavities representing the pigments are created with dielectric constants $\epsilon=1$ inside and $\epsilon=\epsilon_{\mathrm{opt}}=2$ outside the cavities. A Poisson equation is solved for the potential $\phi_{m}(\mathbf{r})$ of the transition charges of pigment $m$ (Adolphs and Renger 2006; Adolphs et al. 2008)

$\nabla\left(\epsilon(\mathbf{r}) \nabla \phi_{m}(\mathbf{r})\right)=-4 \pi \sum_{I} \tilde{q}_{I}^{(m)}(0,1) \delta\left(\mathbf{r}-\mathbf{R}_{I}^{(m)}\right)$

where the dielectric constant $\epsilon(\mathbf{r})=1$, if $\mathbf{r}$ points inside a pigment cavity, and $\epsilon(\mathbf{r})=n^{2}$ otherwise and $\mathbf{R}_{I}^{(m)}$ denotes the position of the Ith nucleus of this pigment. The excitonic coupling of pigment $m$ with pigment $n$ is then obtained as

$V_{m n}=\sum_{J} \phi_{m}\left(\mathbf{R}_{J}^{(n)}\right) \tilde{q}_{J}^{(n)}(0,1)$,

where $\mathbf{R}_{J}^{(n)}$ is the position of the $J$ th nucleus of pigment $n$ with transition partial charge $\tilde{q}_{J}^{(n)}(0,1)$. The transition charges $\tilde{q}_{I / J}^{(m / n)}(0,1)$ of the pigments contain a correction, as described above (Eq. 30). Finally, we note that the present classical electrostatics approach can be justified by quantum mechanical perturbation theory (Hsu et al. 2001; Renger and Müh 2012).

\section{Critical approximations of Poisson-TrEsp and comparison with other methods}

The approximation of the optical polarization of the protein by that of a homogeneous dielectric has been investigated recently by Curutchet et al. (2011) in the framework of a polarizable force field model that assigns atomic polarizabilities and, in this way, takes into account the heterogeneous polarizability of the PPC. An enhancement of energy transfer rates by as much as a factor of 4 resulted when the calculations were compared with that employing a homogeneous dielectric. Interestingly, an average optical dielectric constant of 2 was obtained, which agrees with the estimates given above.

Another approximation of Poisson-TrEsp concerns the neglect of the polarization of the pigments, by assigning a dielectric constant of one to the interior of the cavities. This approximation is in part justified by the fact that the $0 \rightarrow 1$ transition due to its low excitation energy is a major contributor to the polarization of the pigments and the interaction between $0 \rightarrow 1$ transitions of the pigments is explicitly taken into account as excitonic coupling in the calculation of optical spectra and energy transfer. A promising tool to include also the residual polarization originating from higher excited pigment states is the density-fragment interaction (DFI) approach proposed by Fujimoto and Yang (2008). This method (referred to as transition density fragment interaction (TDFI) method) has recently been applied to calculate excitonic couplings either excluding (Fujimoto and Hayashi 2009; Fujimoto 2010) or including (Fujimoto 2012) interpigment electron exchange. What is still missing is the effect of the polarization of the protein environment. A combination of TDFI with Poisson-TrEsp might be possible.

\section{How far can one simplify the calculations of excitonic couplings?}

The simplest possible approximation is the point-dipole approximation

$V_{m n}=f\left(\frac{\mathbf{d}_{m}^{(10)} \cdot \mathbf{d}_{n}^{(10)}}{R_{m n}^{3}}-3 \frac{\left(\mathbf{d}_{m}^{(10)} \cdot \mathbf{R}_{m n}\right)\left(\mathbf{d}_{n}^{(10)} \cdot \mathbf{R}_{m n}\right)}{R_{m n}^{5}}\right)$

where the transition dipole moment $\mathbf{d}_{m}^{(10)}$ is the first moment of the transition density $\rho_{10}^{(m)}(\mathbf{r})$,

$\mathbf{d}_{m}^{(10)}=\int d \mathbf{r} \rho_{10}^{(m)}(\mathbf{r}) \mathbf{r}$.

The direction of the transition dipole moment of the $S_{0} \rightarrow$ $S_{1}$ transition of chlorin type pigments is known from experiments (Kleima et al. 2000; Georgakopoulou et al. 2002; Linke et al. 2008) and from quantum chemical calculations (e.g., Madjet et al. (2006)) to be oriented in good approximation along the $N_{\mathrm{B}}-N_{\mathrm{D}}$ axis of the pigments. Whereas quantum chemical calculations predict an angle $\beta=0^{\circ}$ between this axis and the transition dipole moment (Madjet et al. 2006), the experimental estimates range from $\beta=4.5^{\circ} \pm 2^{\circ}$ to $\beta=-12^{\circ} \pm 3^{\circ}$, estimated from an analysis of time-resolved anisotropy measurements on the peridinin-Chl $a$ complex and from femtosecond polarization resolved visible pump-infrared probe spectroscopy on Chl $a$ in a d $\mathrm{d}_{8}$-toluene solution (Linke et al. 2008), respectively. Note that, a positive value of $\beta$ refers to rotation toward the $13^{1}$ keto group of Chla. A value of $\beta=7^{\circ}$ was reported for BChl $a$ from simulations of circular dichroism spectra of the LH2 complex of purple bacteria (Georgakopoulou et al. 2002). The magnitude of the vacuum transition dipole moment can be extrapolated from an analysis of the dependence of the integral oscillator strength of this 
transition on the refractive index of the solvent (Knox and Spring 2003), as discussed above.

Before we discuss the validity of the point dipole approximation, we will study a simple model for obtaining the screening/local field correction factor $f$ in Eq. 33. For this purpose, we describe the optical transition of the pigment by a point-dipole $\mathbf{d}$ that is centered in a spherical cavity with dielectric constant $\epsilon=1$ inside the cavity and $\epsilon=\epsilon_{\mathrm{opt}}=n^{2}$ outside. The electrostatic potential $\phi(\mathbf{r})$ of the point-dipole outside the sphere is obtained as (Böttcher, 1973)

$\phi(\mathbf{r})=\frac{3}{2 n^{2}+1} \frac{\mathbf{d} \cdot \mathbf{r}}{r^{3}}$

Hence, it equals the electrostatic potential of a point-dipole in a homogeneous dielectric (i.e., without the sphere) with an effective dipole moment

$\mathbf{d}_{\mathrm{eff}}=\mathbf{d} \frac{3 n^{2}}{2 n^{2}+1}$.

In this way, local field effects arising from the presence of the cavity can be effectively described. Knox and Spring (2003) used this empty cavity model (besides two others) to analyze the dipole strength of the $S_{0} \rightarrow S_{1}$ transition of chlorophyll and bacteriochlorophyll in different solvents and, in this way, inferred the vacuum transition dipole strength.

By considering, in addition to the local field effects, the screening of the Coulomb coupling by the factor $1 / n^{2}$, the overall screening/local field correction factor in Eq. (33) follows as

$f=\frac{1}{n^{2}}\left(\frac{3 n^{2}}{2 n^{2}+1}\right)^{2}=\frac{9 n^{2}}{\left(2 n^{2}+1\right)^{2}}$,

which for the present $n^{2}=2$ gives

$f=0.72$.

Three critical approximations of this estimate are: (i) the transition density is approximated by a point dipole, (ii) a spherical cavity is used for the (rather planar) pigment, and (iii) in the solution of the Poisson equation for the potential $\phi(\mathbf{r})$ the presence of a second cavity next to the original cavity was neglected.

These approximations can be tested by comparison of the resulting $f$ factor to values obtained with the PoissonTrEsp method introduced above. Interestingly, comparison of couplings calculated with Poisson-TrEsp for $\epsilon=2$ with those obtained for $\epsilon=1$ shows that on average $f$ indeed varies between 0.6 (Müh et al. 2012) and 0.8 (Adolphs and Renger 2006) for the different complexes investigated (Adolphs and Renger 2006; Adolphs et al. 2010; Müh et al. 2012; Müh and Renger 2012).
If, on the other hand, vacuum couplings obtained with TrEsp are compared with point-dipole values, large deviations are obtained in the case of photosystem I, and in some cases for LHCII (Müh et al. 2010) and CP43 (Müh et al. 2012). Since, in these cases, despite the failure of the point-dipole approximation, a correct average screeninglocal field correction factor $f$ is predicted by the pointdipole-in-a-spherical-cavity model, we have to conclude that Eq. (37) relies on error compensation between the point-dipole and spherical-cavity approximation. Nevertheless, it seems to work well. Note that, a similar error compensation was found in the calculation of dispersive line shifts in nonpolar solvents discussed above.

The dipolar shape of the transition density (Madjet et al. 2006) suggests a considerable improvement of the pointdipole approximation by an extended dipole approximation. In the latter, the transition density is approximated by just two partial charges of opposite sign that are placed along the direction of the transition dipole moment, at a distance of about $9 \AA$ (Madjet et al. 2006; Renger et al. 2008). Note that this distance is consistent with the estimate resulting from solvation energies discussed above and in Renger et al. (2008). The excitonic coupling then results from a sum over four Coulomb interactions between two pigments. Indeed, often the failure of the point-dipole approximation can be removed by using an extended dipole approximation (Madjet et al. 2006; Müh et al. 2012). A notable exception is photosystem I (Adolphs et al. 2010), where a description in terms of atomic transition partial charges is indispensable and for some pigments also additional contributions from electron exchange (Madjet et al. 2009) can be expected. In summary, we may conclude that a minimal scheme for the calculation of excitonic couplings in PPCs is the extended dipole approximation (with a dipole extend of about $9 \AA$ for Chls and BChls and the absolute amount of the transition charge chosen such that the experimental vacuum transition dipole moment results) and Eq. (37) for the screening/local field correction factor, resulting in $f \approx 0.7$.

The site energy funnel in the FMO protein

As an example for a successful application of the above calculation schemes of excitonic couplings and site energies, we discuss light-harvesting in the FMO protein. The FMO protein connects the outer antenna system, the chlorosomes, with the reaction center complex in green sulfur bacteria (Fig. 4). Despite its relatively simple structure, as compared to, e.g., the core antennae of PSII (lower part of Fig. 1), it took more than 20 years after its crystal structure was published (Fenna and Matthews 1975) until a site energy set was found that allowed for a quantitative simulation of its linear absorbance as well as linear 
and circular dichroism spectra (Louwe et al. 1997). The key was the assumption of a much lower effective dipole strength of the pigments, than used before, for the calculation of excitonic couplings, by Aartsma and coworkers (Louwe et al. 1997; Wendling et al. 2002).

The proof of the validity of the point dipole approximation used for the calculations of the couplings and a microscopic explanation of the low effective dipole strength was provided by Poisson-TrEsp calculations (Adolphs and Renger 2006), using the vacuum dipole strength of BChla inferred by Knox and Spring (2003). This finding and the confirmation of the site energies of Aartsma and coworkers by a fit of optical spectra using an improved line shape theory and a genetic algorithm led to the prediction of the relative orientation of the FMO protein relative to the RC complex (Adolphs and Renger 2006). The proposed orientation was experimentally verified 3 years later by using a combination of chemical labeling and mass spectrometry by Blankenship and coworkers (Wen et al. 2009).

A first successful verification of the fitted site energies by independent structure-based calculations was achieved by PBQC calculations (Müh et al. 2007) which uncovered the molecular mechanisms of the site energy shifts. It was found that the electric field of the backbone of two alpha helices has a large contribution to the creation of an energy sink at $\mathrm{BChl} 3$.

An eighths pigment was (re)discovered to be bound at the periphery towards the outer chlorosomes in every monomeric subunit of the trimeric FMO protein (Tronrud et al. 2009). The position of this additional pigment, which most likely is lost in standard biochemical preparation techniques of isolated FMO complexes, led Blankenship and co-workers to suggest that this pigment is the linker for energy transfer from the chlorosomes through the intermediate baseplate (Fig. 4, upper part). Full support for this suggestion was obtained from CDC calculations (Schmidt am Busch et al. 2011), which found that BChl 8 is the most blue-shifted pigment in the FMO protein and thereby completes the excitation energy funnel created by the pigment-protein coupling in this PPC. The origin of the large blue shift of BChl 8 was found to be the interaction of this pigment with charged amino acid residues located at the surface of the FMO protein. It was found also that changing the dielectric environment from that of water (in isolated FMO proteins) to that of a neighboring protein, corresponding to in vivo dielectric conditions, only changes the protonation states of the protein so slightly that BChl 8 remains the most blue-shifted pigment.

By taking into account the optical properties of the baseplate and assuming that the baseplate pigments couple predominantly to $\mathrm{BChl} 8$, the transfer from the baseplate through the FMO protein was simulated (Schmidt am

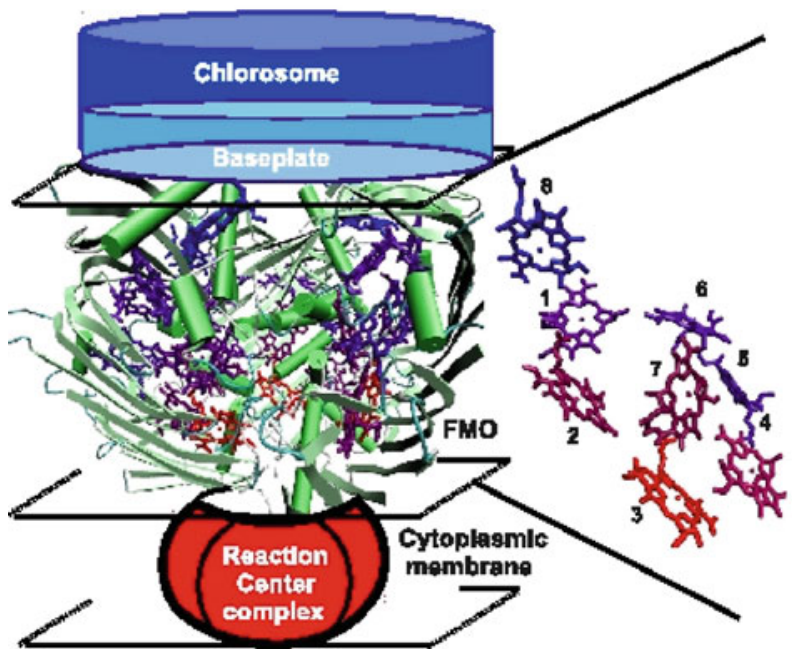

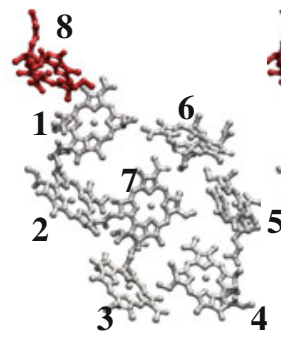

ofs

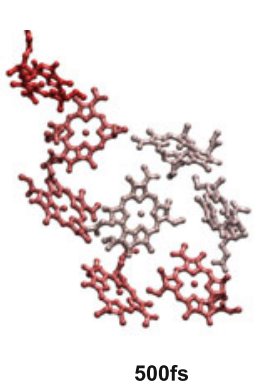

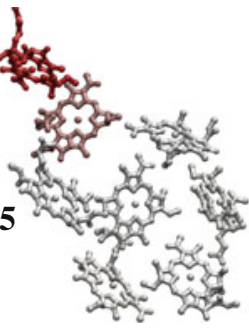

$100 \mathrm{fs}$

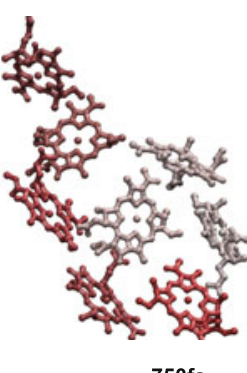

$750 \mathrm{fs}$

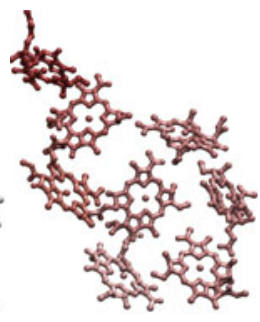

250fs
Fig. 4 Upper part Illustration of the FMO protein and its location between the baseplate/chlorosome and the reaction center complex in green sulfur bacteria. The macrocycles of the bacteriochlorophyll $a$ pigments of one monomeric subunit are shown separately as well and are colored according to their site energy shift, where blue and red mean blue and red shifts, respectively. Lower part Illustration of exciton relaxation through the FMO protein, where the initial excitation is assumed to arrive from the baseplate. The macrocycles of the pigments are colored according to the population of their excited state at a given time

Busch et al. 2011). Generalized Förster theory was used to describe the transfer from the baseplate to the FMO protein and modified Redfield theory for exciton relaxation in the FMO protein. Exciton relaxation was found to occur along a sub 500 fs fast pathway involving pigments 8, 1, 4-7 and 
along a slower 2.5 ps pathway with pigments 1-3 (Fig. 4, lower part). By exciting the FMO protein with ultrafast laser pulses, excitonic/vibronic wavepackets may be created. Excitonic wavepackets have first been detected (Savhikin et al. 1997) and simulated (Renger and May 1998) in the anisotropy of pump-probe spectra. Coherent exciton motion got into the focus of intense research after 2D spectroscopy also revealed these quantum beats (Engel et al. 2007). A fundamental discussion has started about the physiological relevance of these coherences and about the active role the protein might play in protecting these coherences, e.g., by a correlated modulation of site energies. To investigate these correlations in site energy fluctuations, QM/MM approaches were applied (Olbrich et al. 2011a; Jing et al. 2011) but did not find any. It was argued, however, that longer simulation times might be needed to resolve them. Indeed, a normal mode analysis (NMA) revealed that these correlations are particularly strong at very low frequencies (Renger et al. 2012). An inclusion of these correlations in the calculation of exciton relaxation, however, did practically give the same result as a simulation without including them. Hence, we may conclude that these correlations are physiologically not significant. However, the interpretation of 2D spectra critically depends on such correlations (Christensson et al. 2012; Caycedo-Soler et al. 2012). Further below, we will discuss how the spectral density of the pigment-protein coupling can be obtained from a NMA.

\section{Short-range contributions to site energy shifts and excitonic couplings}

If there is considerable wavefunction overlap between neighboring pigments, the building block partition used so far has to be modified. For electron exchange in pigment dimers, quantum chemical calculations on whole dimers and those on the isolated monomers can be related by an effective two-state Hamiltonian (Madjet et al. 2009)

$$
\tilde{\mathbf{H}}_{2}=\left(\begin{array}{cc}
\epsilon_{1}^{(0)}+\lambda_{1}^{(\mathrm{LR})}-\lambda_{1}^{(\mathrm{SR})} & V_{\mathrm{LR}}+V_{\mathrm{SR}} \\
V_{\mathrm{LR}}+V_{\mathrm{SR}} & \epsilon_{2}^{(0)}+\lambda_{2}^{(\mathrm{LR})}-\lambda_{2}^{(\mathrm{SR})}
\end{array}\right),
$$

that contains in the diagonal the local transition energy $\epsilon_{i}^{(0)}$ obtained from quantum chemical calculations on the isolated monomers $i=1,2$ that is shifted in the dimer by $\lambda_{i}^{(\mathrm{LR})}$ due to long-range electrostatic coupling and by $-\lambda_{i}^{(\mathrm{SR})}$ due to electron exchange. (Note that the minus sign on the latter was introduced because the short-range effects are expected to lead to redshifts of the transition energies of the monomers.) The off-diagonal contains long- $\left(V_{\mathrm{LR}}\right)$ and short-range $\left(V_{\mathrm{SR}}\right)$ contributions to the excitonic coupling between the monomers. The long-range contributions $V_{\mathrm{LR}}$ and $\lambda_{i}^{(\mathrm{LR})}$ are obtained from TrEsp and CDC calculations, respectively, introduced above. In order to decipher the short-range contributions, it is necessary to exploit the quantum chemical results on the two lowest excitation energies $E_{+}$and $E_{-}$of the dimer and the corresponding transition dipole moments for the transitions from the ground state, $\boldsymbol{\mu}_{0 \pm}$. The result for the short-range contributions reads (Madjet et al. 2009)

$\lambda_{1,2}^{(\mathrm{SR})}=\epsilon_{1,2}^{(0)}+\lambda_{1,2}^{(\mathrm{LR})}-\frac{1}{2}\left(E_{+}+E_{-}\right) \pm \frac{1}{2}\left(E_{+}-E_{-}\right) \frac{Y^{2}-1}{Y^{2}+1}$,

and

$V_{\mathrm{SR}}=\left(E_{+}-E_{-}\right) \frac{Y}{1+Y^{2}}-V_{\mathrm{LR}}$.

The quantity $Y$ is obtained from the transition dipole moments of the monomers $\boldsymbol{\mu}_{0 i},(i=1,2)$ (obtained from respective quantum chemical calculations) and that of the dimer, $\boldsymbol{\mu}_{0 \pm}$ as

$Y=-h \frac{g_{1}\left(\boldsymbol{\mu}_{0-}\right)}{g_{1}\left(\boldsymbol{\mu}_{0+}\right)}=Y_{a}$.

A second relation for $Y$ is obtained as:

$Y=h \frac{g_{2}\left(\boldsymbol{\mu}_{0+}\right)}{g_{2}\left(\boldsymbol{\mu}_{0-}\right)}=Y_{b}$.

The functions $g_{1}$ and $g_{2}$ are given as

$g_{1}(\boldsymbol{\mu})=\left(\boldsymbol{\mu} \cdot \boldsymbol{\mu}_{01}\right) \boldsymbol{\mu}_{02}^{2}-\left(\boldsymbol{\mu} \cdot \boldsymbol{\mu}_{02}\right)\left(\boldsymbol{\mu}_{01} \cdot \boldsymbol{\mu}_{02}\right)$,

$g_{2}(\boldsymbol{\mu})=\left(\boldsymbol{\mu} \cdot \boldsymbol{\mu}_{02}\right) \boldsymbol{\mu}_{01}^{2}-\left(\boldsymbol{\mu} \cdot \boldsymbol{\mu}_{01}\right)\left(\boldsymbol{\mu}_{01} \cdot \boldsymbol{\mu}_{02}\right)$,

and

$h=1 \quad$ if $\quad g_{1}\left(\boldsymbol{\mu}_{0+}\right) g_{2}\left(\boldsymbol{\mu}_{0-}\right)>0, \quad$ and $\quad h=-1$ otherwise.

The equality of $Y_{a}$ (Eq. 42) and $Y_{b}$ (Eq. 43) can be used as a consistency check of the method. This consistency check became possible by including the transition dipole moments in the analysis, extending an earlier method by Scholes et al. (1999), where only excitation energies were considered.

So far, this method has been applied to the reaction center of purple bacteria (bRC) and PSI (Madjet et al. 2009), revealing that about $3 / 4$ of the excitonic coupling in these special pairs is due to electron exchange and that an additional red shift of the excitation energies of the special pairs due to site energy shifts, also dominated by shortrange effects, results. Here, we report first results on the special pair of PSII that are based on recent crystal structure data (Umena et al. 2011). A constraint geometry optimization was performed, where the torsional angles were kept fixed as in the crystal structure, using density 
functional theory (DFT) with the B3LYP exchange-correlation $(\mathrm{XC})$ functional and a $6-31 \mathrm{G}^{*}$ basis set. Based on this structure, excitation energies and transition dipole moments were calculated for the dimer and the isolated monomers using the different hybrid XC-functionals described in Madjet et al. (2009). The smallest deviation between $Y_{a}$ and $Y_{b}$ was obtained for the B3LYP XCfunctional, resulting in short-range site energy shifts $-\lambda_{1}^{(\mathrm{SR})}\left(Y_{a}\right)=-298 \mathrm{~cm}^{-1}$ and $-\lambda_{1}^{(\mathrm{SR})}\left(Y_{b}\right)=-177 \mathrm{~cm}^{-1}$ for monomer 1 and $-\lambda_{2}^{(\mathrm{SR})}\left(Y_{a}\right)=-325 \mathrm{~cm}^{-1}$ and $-\lambda_{2}^{(\mathrm{SR})}\left(Y_{b}\right)=-150 \mathrm{~cm}^{-1}$ for monomer 2 . The short-range coupling was obtained as $V_{\mathrm{SR}}\left(Y_{a}\right)=146 \mathrm{~cm}^{-1}$ and $V_{\mathrm{SR}}\left(Y_{b}\right)=65 \mathrm{~cm}^{-1}$. The respective long-range site energy shifts are $\lambda_{1}^{(\mathrm{LR})}=206 \mathrm{~cm}^{-1}$ and $\lambda_{2}^{(\mathrm{LR})}=51 \mathrm{~cm}^{-1}$, and the long-range excitonic couplings, rescaled such as to reflect an effective dipole strength of $4.38 \mathrm{D}$ (Madjet et al. 2009) is $V_{\mathrm{LR}}^{\text {(eff) }}=-26 \mathrm{~cm}^{-1}$. An illustration of these results is given in Fig. 2. Interestingly, the short- and long-range site energy shifts in PSII have opposite sign, whereas in bRC they have the same sign. In addition, the absolute magnitude is much smaller in PSII, and there is a sign flip between the long- and short-range excitonic couplings, indicating an inversion of the redistribution of oscillator strength. The different signs of short- and long-range site energy shifts and the overall smaller magnitude of short-range effects in PSII as compared to bRC explain the much shallower energy trap formed in the special pair of the former (Fig. 2). Finally, we note that the inverted redistribution of oscillator strength in the special pair of PSII is an interesting result that needs to be studied in more detail in the calculation of optical properties. We note, however, that the relative large deviation between $Y_{a}$ and $Y_{b}$ (and the resulting short-range site energy shifts and excitonic couplings) does not allow to draw final conclusions yet. This deviation could have at least two reasons: (i) limitations of the quantum chemical method and (ii) limitations of the effective two-state model used to analyze the quantum chemical results.

\section{The exciton-vibrational Hamiltonian and the spectral density derived from a normal mode analysis}

The exciton Hamiltonian $H_{\text {ex }}$ has been derived by assuming that the nuclear degrees of freedom of the PPC are fixed at their equilibrium positions in the electronic ground state. The displacements of nuclei from these positions result in a change of the transition energies and excitonic couplings that is described by the exciton-vibrational Hamiltonian $H_{\text {ex-vib. Hence, we may write }}$

$H_{\mathrm{ex}}+H_{\mathrm{ex}-\mathrm{vib}}=\sum_{m, n} H_{m n}(\mathbf{R})|m\rangle\langle n|$ where the nuclear degrees of freedom are abbreviated by $\mathbf{R}$ and where for the equilibrium positions $\mathbf{R}^{(0)}$ the exciton matrix

$H_{m n}^{(0)}=H_{m n}\left(\mathbf{R}^{(0)}\right)=\delta_{m n} E_{m}+\left(1-\delta_{m n}\right) V_{m n}$

contains in the diagonal the site energies $E_{m}$ (Eq. 17) and in the off-diagonal the excitonic couplings $V_{m n}$ (Eqs. 11, 19)derived above.

We will in the following consider changes of these quantities that occur for small displacements of nuclei from their equilibrium positions. By using a Taylor expansion of the exciton matrix $H_{m n}(\mathbf{R})$ we have

$H_{m n}(\mathbf{R}) \approx H_{m n}^{(0)}+\sum_{J}\left(\left.\nabla_{J} H_{m n}\right|_{0}\right) \cdot\left(\mathbf{R}_{J}-\mathbf{R}_{J}^{(0)}\right)$,

where $\left(\left.\nabla_{J} H_{m n}\right|_{0}\right)$ is the gradient of $H_{m n}$ taken with respect to the three Cartesian coordinates of atom $J$ at the equilibrium position of nuclei in the electronic ground state of the PPC.

In the case of small displacements of nuclei, a normal mode analysis may be used to describe the nuclear dynamics in the electronic ground state of the PPC. Mass weighted dimensionless normal coordinates $Q_{\xi}$ are introduced via

$\mathbf{R}_{J}-\mathbf{R}_{J}^{(0)}=\frac{\hbar}{\left(2 M_{J}\right)^{1 / 2}} \sum_{\xi} \mathbf{A}_{J}^{(\xi)} \frac{Q_{\xi}}{\omega_{\xi}^{1 / 2}}$

where $M_{J}$ is the mass of atom $J$ and $\mathbf{A}_{J}^{(\xi)}$ contains the contributions of this atom to the eigenvector of normal mode $\xi$.

By using the CDC expression (Eq. 22) for the coordinate dependence of the site energies and the TrEsp expression (Eq. 28) for that of the excitonic couplings, the gradient $\nabla_{J} H_{m n}$ in Eq. (49) can be calculated and the exciton-vibrational coupling Hamiltonian is obtained as (Renger et al. 2012)

$H_{\mathrm{ex}-\mathrm{vib}}=\sum_{\xi} \sum_{m n} \hbar \omega_{\xi} g_{\xi}(m, n) Q_{\xi}|m\rangle\langle n|$,

where the dimensionless coupling constant $g_{\xi}(m, n)$ was introduced as

$$
\begin{aligned}
& \omega_{\xi}^{3 / 2}(2 \hbar)^{1 / 2} g_{\xi}(m, n) \\
& =\frac{\delta_{m n}}{\epsilon_{\mathrm{eff}}} \sum_{I, J, a} \frac{q_{J}^{(a)}\left(q_{I}^{(m)}(1,1)-q_{I}^{(m)}(0,0)\right)}{\left|\mathbf{R}_{J, a}^{(0)}-\mathbf{R}_{I, m}^{(0)}\right|^{3}} \\
& \quad \times\left(\mathbf{R}_{J, a}^{(0)}-\mathbf{R}_{I, m}^{(0)}\right) \cdot\left(M_{I}^{-1 / 2} \mathbf{A}_{I}^{(\xi)}-M_{J}^{-1 / 2} \mathbf{A}_{J}^{(\xi)}\right) \\
& \quad+\left(1-\delta_{m n}\right) f \sum_{I, J} \frac{q_{I}^{(m)}(0,1) q_{J}^{(n)}(0,1)}{\left|\mathbf{R}_{I, m}^{(0)}-\mathbf{R}_{J, n}^{(0)}\right|^{3}} \\
& \quad \times\left(\mathbf{R}_{I, m}^{(0)}-\mathbf{R}_{J, n}^{(0)}\right) \cdot\left(M_{J}^{-1 / 2} \mathbf{A}_{J}^{(\xi)}-M_{I}^{-1 / 2} \mathbf{A}_{I}^{(\xi)}\right) .
\end{aligned}
$$


From the above coupling constants and the density of vibrational states, both obtained from the NMA, the spectral density of the exciton-vibrational coupling

$J_{m n k l}(\omega)=\sum_{\xi} g_{\xi}(m, n) g_{\xi}(k, l) \delta\left(\omega-\omega_{\xi}\right)$

can be calculated, which is a key quantity in the calculation of optical spectra and excitation energy transfer [see, e.g., a recent review by (Renger and Müh 2013)]. It describes the fluctuation of site energies $(m=n=k=l)$ the fluctuation of excitonic couplings $(m=k, n=l, m \neq n)$, the correlations in fluctuations of site energies $(m=n, k=l$, $m \neq k$ ), and also correlations between fluctuations of site energies and excitonic couplings and between fluctuations of excitonic couplings.

This method has been applied to the monomeric subunit of the FMO protein (Renger et al. 2012). The main results of this analysis are: (i) the fluctuation of site energies (Fig. 5) are an order of magnitude stronger than the fluctuations in excitonic couplings. (ii) Although the correlations in site energy fluctuations (Fig. 6) are of similar magnitude as the site energy fluctuations, the former are particularly large at low frequencies and, as noted before, these correlations have practically no influence on exciton relaxation or the decay of coherences between different exciton states. They might, however, be important for the interpretation of $2 \mathrm{D}$ spectra. The comparison of the different $J_{m m m m}(\omega)$ characterizing the site energy fluctuations of pigments $m=1 \ldots 7$ with experimental quantities resulting from fluorescence line narrowing spectroscopy shows that the NMA spectral densities seem to be larger than the experimental quantities at low frequencies and smaller at higher frequencies. This systematic deviation might have its origin in the neglect of anharmonicities of the force field (in particular the non-bonding interaction) in the NMA. We are currently performing molecular dynamics simulations to investigate this effect.

Finally, we note that earlier QM/MM approaches (Damjanovic et al. 2002; Olbrich et al. 2011b; Shim et al. 2012), where QC calculations of pigment transition energies were performed along the classical trajectories of nuclei obtained from MD simulations, did not obtain agreement with experimental spectral densities. Most likely the reason is the mismatch between nuclear geometries resulting from a classical force field and from QC calculations, taking into account that $\mathrm{QC}$ excitation energies are very sensitive to slight geometry distortions.

\section{The quantum dynamics of excitons}

In order to describe energy transfer and lineshape functions measured in optical experiments, it is necessary to include nuclear dynamics into the theory. In the spirit of the BornOppenheimer approximation, suitable potential energy surfaces (PES) for the motion of nuclei are introduced, where the choice of the PES depends on the relative strength of excitonic and exciton-vibrational coupling. If relaxation of nuclei within these PES is fast as compared to the transfer between different PES, perturbation theory in the inter-PES coupling can be used to derive expressions for rate constants.

To construct such PES, we consider the Hamiltonian parameterized above

$H=H_{\mathrm{ex}}+H_{\mathrm{ex}-\mathrm{vib}}+H_{\mathrm{vib}}$

with the exciton Hamiltonian $H_{\text {ex }}$ (Eq. 20), the excitonvibrational coupling Hamiltonian $H_{\text {ex-vib }}$ (Eq. 51) and the vibrational Hamiltonian

$H_{\text {vib }}=\sum_{\xi} \frac{\hbar \omega_{\xi}}{4}\left(P_{\xi}^{2}+Q_{\xi}^{2}\right)\left(\sum_{m}|m\rangle\langle m|+| 0\rangle\langle 0|\right)$

where we used the completeness relation $1=$ $\sum_{m}|m\rangle\langle m|+| 0\rangle\langle 0|$ to introduce a unity operator on the r.h.s. of Eq. (55) (restricting the electronic Hilbert space to the ground state $|0\rangle$ and first excited states $|m / n\rangle$ of the PPC) and introduced dimensionless normal coordinates $Q_{\xi}$ and momenta $P_{\xi}$. The frequencies of the normal modes are $\omega_{\xi}$. Hence, the full PPC Hamiltonian may be written as

$$
\begin{aligned}
H= & \sum_{m, n}\left\{\delta_{m, n}\left(E_{m}+\sum_{\xi} \frac{\hbar \omega_{\xi}}{4} Q_{\xi}^{2}\right)\right. \\
& \left.+\sum_{\xi} \hbar \omega_{\xi} g_{\xi}(m, n) Q_{\xi}+\left(1-\delta_{m, n}\right) V_{m n}\right\}|m\rangle\langle n| \\
& +\sum_{\xi} \frac{\hbar \omega_{\xi}}{4} Q_{\xi}^{2}|0\rangle\langle 0|+T_{\text {nucl }}
\end{aligned}
$$

where $T_{\text {nucl }}=\sum_{\xi} \frac{\hbar \omega_{\xi}}{4} P_{\xi}^{2}$ is the kinetic energy of nuclei. This Hamiltonian is used in the following to consider some limiting cases, in which expressions for rate constants of energy transfer can be derived.

Supported by the NMA-derived spectral density discussed above, we assume, that the fluctuation of excitonic couplings is much smaller than the fluctuation of site energies and, therefore, the former can be neglected. The Hamiltonian of single excited electronic states then becomes

$$
\begin{aligned}
H_{1}= & \sum_{m}\left(E_{m}+\sum_{\xi}\left(\frac{\hbar \omega_{\xi}}{4} Q_{\xi}^{2}+\hbar \omega_{\xi} g_{\xi}(m, m) Q_{\xi}\right)\right) \\
& \times|m\rangle\left\langle m\left|+\sum_{m, n}^{m \neq n} V_{m n}\right| m\right\rangle\langle n|+T_{\text {nucl }}
\end{aligned}
$$

In the spirit of the Born-Oppenheimer approximation, we have to diagonalize the electronic Hamiltonian in order to obtain the PES for the motion of nuclei. If the difference in 

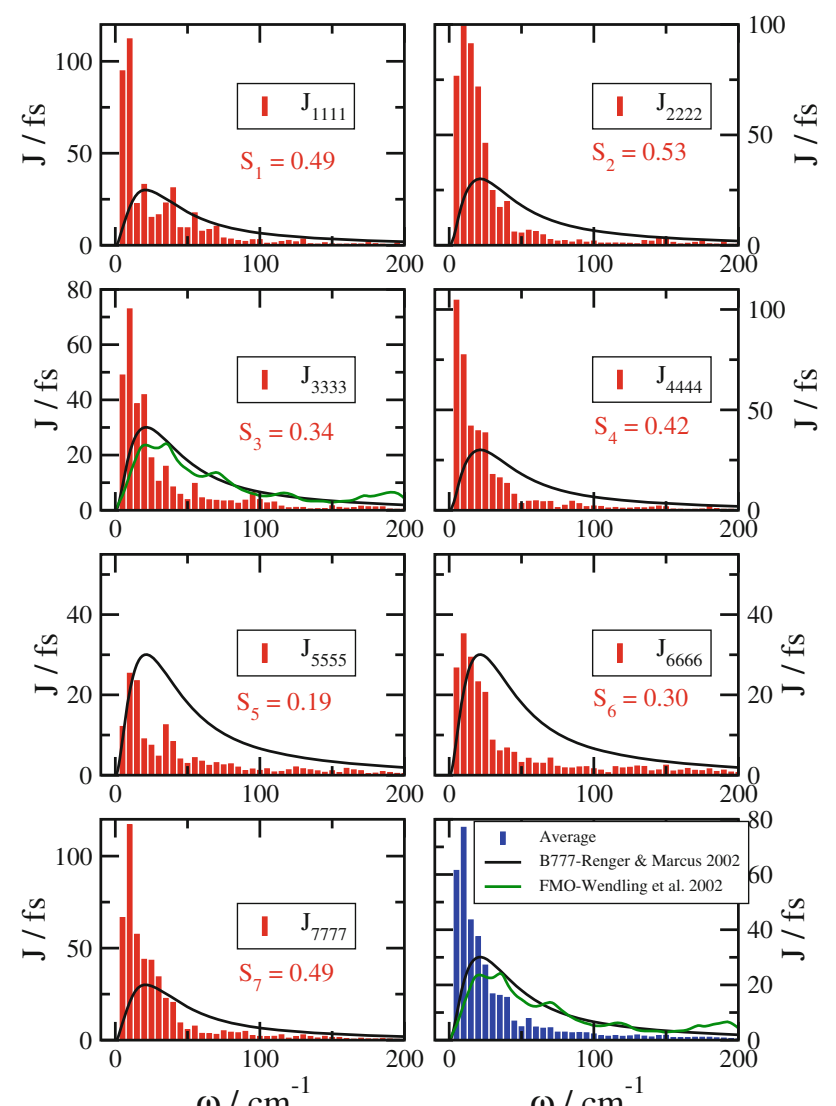

Fig. 5 Spectral density $J_{\text {mmmm }}(\omega)$ containing the site energy fluctuations of pigments $m=1 . .7$ of the monomeric subunit of the FMO protein, shown as red bars, obtained from a normal mode analysis (Renger et al. 2012). The red numbers are the resulting Huang-Rhys factors $S_{m}=\sum_{\xi} g_{\xi}(m, m)^{2}$. The black solid line denotes the spectral density of the B777 complex, extracted (Renger and Marcus 2002) from fluorescence line narrowing spectra (Creemers et al. 1999) (the Huang-Rhys factor has been varied from $S=1.3$ for B777 to $S=0.42$ for FMO in order to describe the temperature dependence of absorbance spectra of the FMO protein). In the right lower corner the average spectral density of the seven sites (blue bars) is compared to the experimental quantity of the B777 complex and the spectral density obtained from fluorescence line narrowing spectra of the FMO protein (Wendling et al. 2000)

diagonal elements of the above matrix are much larger than the excitonic couplings in the off-diagonal, the resulting eigenstates may be well approximated by the localized excited states and the nuclei, therefore effectively move in the PES $U_{m}$, resulting from the above diagonal elements as

$U_{m}=U_{m}^{(0)}+\sum_{\xi} \frac{\hbar \omega_{\xi}}{4}\left(Q_{\xi}+2 g_{\xi}(m, m)\right)^{2}$

where the minimum in energy of this PES is at

$U_{m}^{(0)}=E_{m}-\sum_{\xi} \hbar \omega_{\xi} g_{\xi}(m, m)^{2}$

In this case, localized excited states are created in the PPC, the nuclei relax fast in the above PES and a second
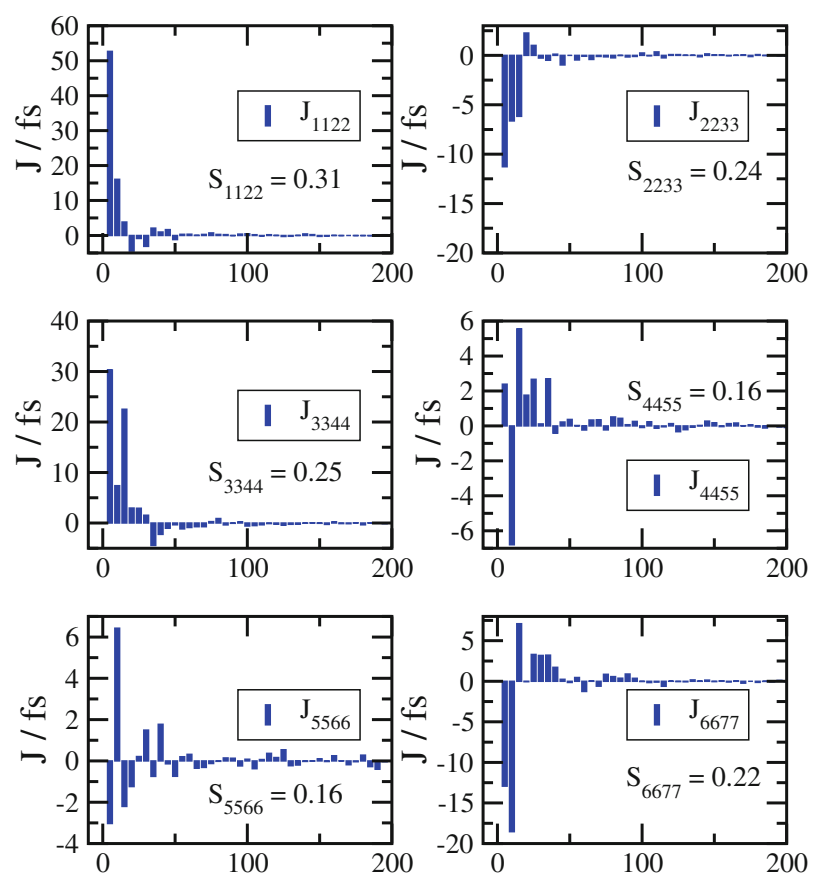

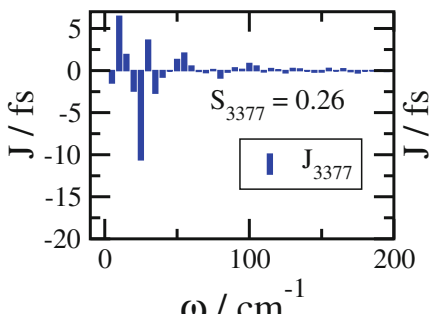

$\omega / \mathrm{cm}^{-1}$

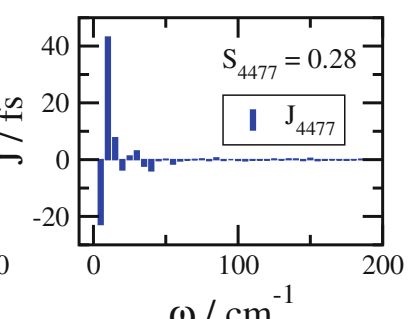

$\omega / \mathrm{cm}^{-1}$
Fig. 6 Spectral density $J_{m m n n}(\omega)$ containing the correlation in site energy fluctuations between pigments $m$ and $n$ of the monomeric subunit of the FMO protein, shown as blue bars, obtained from a normal mode analysis. The numbers are generalized Huang-Rhys factors $S_{m m n n}=\sum_{\xi}\left|g_{\xi}(m, m) g_{\xi}(n, n)\right|$. The correlations of those pigment pairs with the largest correlation in site energy fluctuations (i.e., the largest generalized Huang-Rhys factors) are shown

order perturbation theory in the small inter-PES coupling $V_{m n}$ results in the Förster rate constant of excitation energy transfer between states $|m\rangle$ and $|n\rangle$, if, in addition, correlations in site energy fluctuations are neglected. A detailed derivation of this rate constant was given, e.g., in a recent review by Renger and Müh (2013).

In the case of stronger excitonic coupling, the PES will change. For illustrative purposes we will consider a molecular dimer first and take into account just one vibrational coordinate $Q$ with vibrational frequency $\omega$. In this case, the Hamiltonian can be diagonalized resulting in the PES $U_{M}(Q)$ of adiabatic states (eigenstates)

$$
\begin{aligned}
U_{M}(Q)= & \frac{1}{2}\left[E_{1}+E_{2}+2 \hbar \omega \bar{g} Q+\frac{\hbar \omega}{2} Q^{2}\right] \\
& \pm \frac{1}{2} \sqrt{[(\Delta E+\hbar \omega \Delta g Q)]^{2}+4 V_{12}^{2}}
\end{aligned}
$$


where $\Delta E=E_{1}-E_{2}, \Delta g=g(1,1)-g(2,2)$, and

$\bar{g}=(g(1,1)+g(2,2)) / 2$.

For small differences in exciton-vibrational coupling, i.e., for $\hbar \omega \Delta g<V_{12}$, a Taylor expansion may be used to approximate the above PES by

$$
\begin{aligned}
U_{M}(Q) \approx & \frac{1}{2}\left[E_{1}+E_{2}+2 \hbar \omega \bar{g} Q+\frac{\hbar \omega}{2} Q^{2}\right] \\
& \pm \frac{1}{2}\left(\sqrt{\Delta E^{2}+4 V_{12}^{2}}+\frac{\Delta E \Delta g}{\sqrt{\Delta E^{2}+4 V_{12}^{2}}} \hbar \omega Q\right) .
\end{aligned}
$$

Neglecting the last term, which is justified for small $\Delta g$ or small $\Delta E$, then results in PES $U_{M}(Q)=U_{ \pm}(Q)$ which are mutually unshifted along the coordinate axis (Müh and Renger 2013)

$U_{ \pm}(Q) \approx U_{ \pm}^{(0)}+\frac{\hbar \omega}{4}(Q+2 \bar{g})^{2}$

where the minima in energy are at

$U_{ \pm}^{(0)}=\frac{1}{2}\left[E_{1}+E_{2}-2 \bar{g}^{2} \hbar \omega \pm \sqrt{\Delta E^{2}+4 V_{12}^{2}}\right]$

and the shift with respect to the PES of the electronic ground state is determined by $\bar{g}$ for both PES. In this case one can assume that after optical excitation, the nuclei relax in the initial PES and no further reorganization of nuclei occurs upon exciton transfer, as in Redfield theory discussed below.

For larger $\Delta g$ and $\Delta E \neq 0$, the last term in Eq. (62) needs to be taken into account and the PES

$U_{ \pm}(Q) \approx \tilde{U}_{ \pm}^{(0)}+\frac{\hbar \omega}{4}\left(Q+2\left(\bar{g}+g_{ \pm}\right)\right)^{2}$

of the exciton states get displaced with respect to each other due to

$g_{ \pm}= \pm \frac{\Delta E \Delta g}{2 \sqrt{\Delta E^{2}+4 V_{12}^{2}}}$

and we have

$\tilde{U}_{ \pm}^{(0)}=U_{ \pm}^{(0)}-\hbar \omega\left(2 \bar{g}+g_{ \pm}\right) g_{ \pm}$.

Now, after nuclear relaxation in the PES of the initially excited PES, exciton transfer occurs between displaced PES and, hence, a nuclear reorganization needs to be taken into account as in Modified Redfield theory discussed below.

We return now to the case of many pigments and many vibrational normal modes of the PPC coupling to the optical transitions of the pigments. Exciton states

$|M\rangle=\sum_{m} c_{m}^{(M)}|m\rangle$ are introduced as eigenstates of $H_{1}$ (Eq. 57) for the equilibrium position of nuclei in the electronic ground state, i.e., for $Q=0$. Hence, the exciton matrix $H_{m n}^{(0)}(\mathrm{Eq} .48)$ is diagonalized.

Transforming the Hamiltonian (Eq. 56) into the new basis results in

$$
\begin{aligned}
H= & \sum_{M}\left(\mathcal{E}_{M}+\sum_{\xi} \frac{\hbar \omega_{\xi}}{4} Q_{\xi}^{2}\right)|M\rangle\langle M| \\
& +\sum_{M, N} \sum_{\xi} \hbar \omega_{\xi} g_{\xi}(M, N) Q_{\xi}|M\rangle\langle N| \\
& +\sum_{\xi} \frac{\hbar \omega_{\xi}}{4} Q_{\xi}^{2}|0\rangle\langle 0|+T_{\text {nucl }}
\end{aligned}
$$

where the exciton-vibrational coupling constants in the new basis read

$g_{\xi}(M, N)=\sum_{m n} c_{m}^{(M)} c_{n}^{(N)} g_{\xi}(m, n)$.

The off-diagonal elements $\hbar \omega_{\xi} g_{\xi}(M, N) Q_{\xi}|M\rangle\langle N|(M \neq$ $N)$ couple different exciton states. A second-order perturbation theory in this coupling is used in Redfield and Modified Redfield theory to derive expressions for rate constants of exciton transfer. The diagonal part $\hbar \omega_{\xi} g_{\xi}(M, M) Q_{\xi}|M\rangle\langle M|$ of the exciton-vibrational coupling in $H$ describes the shift along the coordinate axes of the PES of different exciton states with respect to the PES of the ground state. In Redfield theory, the resulting mutual shifts of the PES of different exciton states are neglected, whereas in Modified Redfield theory, these shifts are taken into account in the derivation of a rate constant for exciton transfer. For a recent review on this topic, see, e.g., Renger and Müh (2013).

In both theories, it is assumed that after optical excitation there is first relaxation within the excitonic PES, before exciton relaxation occurs to a different exciton state. Such an assumption requires the diagonal parts of the exciton-vibrational coupling to be large as compared to the off-diagonal parts. There is indeed support for this assumption from a recent normal mode analysis of the coupling constants of the FMO protein (Renger et al. 2012).

In principle, both, the electronic and the electronvibrational coupling can be treated non-perturbatively (Ishizaki and Fleming 2009; Chin et al. 2010; Nalbach et al. 2011; Kreisbeck and Kramer 2012). However, the numerical effort is considerable and, therefore, these methods so far can only be applied to small systems containing a few pigments. An alternative, but numerically also very costly approach includes selected vibrational coordinates explicitly in the system dynamics and uses perturbation theory for the coupling of these coordinates to 
the remaining coordinates which form a heat bath (Kühn et al. 1996; Novoderezhkin et al. 2004).

\section{Conclusions and challenges for future work}

We have given an overview of structure-based modelling of light-harvesting in photosynthesis. We believe that the dominant contribution to site energy shifts, excitonic couplings, and spectral densities comes from the charge- and transition-density Coulomb couplings between the building blocks of the PPC. ${ }^{1}$ Our QC/electrostatic two-step procedure, originally developed for the FMO protein, has been successfully applied to larger complexes like LHCII, CP43 and photosystem I, as reviewed recently (Renger and Müh 2013). A challenge for future work will be to quantify the contributions that have so far been neglected, like dispersive site energy shifts (Heinz et al. 2001; Renger et al. 2008; Ikabata and Nakai 2012), the effect of different conformations of the pigments on the site energies (Zucchelli et al. 2012), the effect of the residual polarization of the pigments on the excitonic interaction (Fujimoto and Yang 2008), and the effect of anharmonic nuclear motion on the spectral density of the pigment-protein coupling (Rivera et al. 2013). A challenge for the development of dynamical theory is the application of non-perturbative approaches (Ishizaki and Fleming 2009; Chin et al. 2010; Nalbach et al. 2011; Kreisbeck and Kramer 2012) to large systems, in order to describe a dynamic localization of exciton states and the formation of exciton domains. It will be interesting to see the limitations of modified Redfield and generalized Förster theory in this context. Concerning the description of short-range contributions to excitonic couplings and site energy shifts, a challenge is to improve the quantum chemical description of exciton states and their coupling to charge transfer (CT) states (Stein et al. 2009). The next challenge will be to include the protein environment in these calculations in order to obtain more realistic energies, in particular of the CT states. In this context, it will be interesting to look for low-lying CT states in the reaction center of photosystem II inferred by Krausz and co-workers based on experiments, where charge separation at low temperatures was initiated with long-wavelength excitation (Krausz et al. 2005; Hughes et al. 2006).

Finally, we have to note that the physiological function of many PPCs includes photoprotection that most likely is connected with conformational transitions of the protein. Hence, static crystal structure data can only serve as a

\footnotetext{
${ }_{1}$ A notable exception is the class of covalently bound opentetrapyrrole type bilin chromophores in phycobilisomes (MacColl 1998).
}

starting point for modelling these processes, which ultimately need to be understood at the same level of molecular detail as we understand the optical properties and energy transfer of small PPCs, like the FMO protein, now.

Acknowledgments Financial support by the Austrian Science Fund (FWF): P 24774-N27 is gratefully acknowledged.

\section{References}

Adolphs J, Renger T (2006) How proteins trigger excitation energy transfer in the FMO complex of green sulfur bacteria. Biophys $\mathbf{J}$ 91:2778-2797

Adolphs J, Müh F, Madjet ME, Renger T (2008) Calculation of pigment transition energies in the FMO protein: from simplicity to complexity and back. Photosynth Res 95:197-209

Adolphs J, Müh F, Madjet ME, Schmidt am Busch M, Renger T (2010) Structure-based calculations of optical spectra of photosystem I suggest an asymmetric light-harvesting process. J Am Chem Soc 132:3331-3343

Bergström H, van Grondelle R, Sundström V (1989) Characterization of excitation energy trapping in photosynthetic purple bacteria at 77 K. FEBS Lett 250:503-508

Böttcher CJF (1973) Theory of electric polarization. Elsevier, Amsterdam

Caycedo-Soler F, Chin AW, Almeida J, Huelga SF, Plenio M (2012) The nature of the low energy band of the Fenna-MatthewsOlson complex: vibronic signatures. J Chem Phys 136:155,102

Chin AW, Rivas A, Huelga SF, Plenio MB (2010) Exact mapping between system-reservoir quantum models and semi-infinite discrete chains using orthogonal polynomials. J Math Phys 51:092,109

Christensson N, Kauffmann HF, Pullerits T, Mancal T (2012) Origin of long-lived coherences in light-harvesting complexes. J Phys Chem B 116:7449-7454

Creemers TMH, De Caro CA, Visschers RW, van Grondelle R, Völker S (1999) Spectral hole burning and fluorescence line narrowing in subunits of the light-harvesting complex LH1 of purple bacteria. J Phys Chem B 103:9770-9776

Curutchet C, Scholes GD, Mennucci B, Cammi R (2007) How solvent controls electronic energy transfer and light harvesting: toward a quantum-mechanical description of reaction field and screening effects. J Phys Chem B 111:13253-13265

Curutchet C, Kongsted J, Munoz-Losa A, Hossein-Nejad H, Scholes GD, Mennucci B (2011) Photosynthetic light-harvesting is tuned by the heterogeneous polarizable environment of the protein. J Am Chem Soc 133:3078-3084

Damjanovic A, Kosztin I, Kleinekathöfer U, Schulten K (2002) Excitons in a photosynthetic light-harvesting system: a combined molecular dynamics, quantum chemistry, and polaron model study. Phys Rev E 65(1-24):031,919

Engel GS, Calhoun TR, Read EL, Ahn TK, Mancal T, Cheng YC, Blankenship RE, Fleming GR (2007) Evidence for wavelike energy transfer through quantum coherence in photosynthetic systems. Nature 446:782-786

Fenna RE, Matthews BW (1975) Chlorophyll arrangement in a bacteriochlorophyll protein from Chlorobium limicola. Nature 258:573-577

Förster T (1948) Zwischenmolekulare Energiewanderung und Fluoreszenz. Ann Phys Leipzig 2:55-75

Fujimoto KJ (2010) Transition-density-fragment interaction approach for exciton-coupled circular dichroism spectra. J Chem Phys $133: 124101$ 
Fujimoto KJ (2012) Transition-density-fragment interaction combined with transfer integral approach for excitation-energy transfer via charge transfer states. J Chem Phys 137:034101

Fujimoto KJ, Hayashi S (2009) Electronic coulombic coupling of excitation-energy transfer in xanthorhodopsin. J Am Chem Soc $131: 14,152$

Fujimoto KJ, Yang W (2008) Density-fragment interaction approach for quantum-mechanical/molecular-mechanical calculations with application to the excited states of a $\mathrm{Mg}^{2+}$-sensitive dye. J Chem Phys 129:054,102

Georgakopoulou S, Frese R, Johnson E, Koolhaas C, Cogdell RJ, van Grondelle R, van der Zwan G (2002) Absorption and CD spectroscopy and modeling of various LH2 complexes from purple bacteria. Biophys J 82:2184-2197

Heinz H, Suter UW, Leontidis E (2001) Simple and accurate computation of solvatochromic shifts in $\pi \rightarrow \pi^{*}$ transitions of aromatic chromophores. J Am Chem Soc 123:11229-11236

Hsu C, Head-Gordon M, Head-Gordon T, Fleming GR (2001) Excitation energy transfer in condensed media. J Chem Phys 114:3065-3072

Hughes JL, Smith P, Pace R, Krausz E (2006) Charge separation in photosystem II core complexes induced by $690-730 \mathrm{~nm}$ excitation at $1.7 \mathrm{~K}$. Biochim Biophys Acta 4:841-851

Ikabata Y, Nakai H (2012) Extension of local response dispersion method to excited-state calculation based on time-dependent density functional theory. J Chem Phys 137:124,106

Ishizaki A, Fleming GR (2009) Unified treatment of quantum coherent and incoherent hopping dynamics in electronic energy transfer: reduced hierarchy equation approach. J Chem Phys $130: 234,111$

Jang S, Newton MD, Silbey RJ (2004) Multichromophoric Förster resonance energy transfer. Phys Rev Lett 92:218,301

Jing Y, Zheng R, Li HX, Shi Q (2011) Theoretical study of the electronic-vibrational coupling in the $\mathrm{Q}_{\mathrm{y}}$ states of the photosynthetic reaction center in purple bacteria. J Phys Chem B 116:1164-1171

Katiliene Z, Kautilius E, Woodbury NW (2003) Energy trapping and detrapping in reaction center mutants from Rhodobacter sphaeroides. Biophys J 84:3240-3251

Kell A, Feng X, Reppert M, Jankowiak R (2013) On the shape of the phonon spectral density in photosynthetic complexes. J Phys Chem B 117:7317-7323

Kleima FJ, Hofmann E, Gobets B, van Stokkum IHM, van Grondelle R, Diederichs K, van Amerongen H (2000) Förster excitation energy transfer in peridinin-chlorophyll- $a$-protein. Biophys $\mathrm{J}$ 78:344-353

Knox RS, Spring BQ (2003) Dipole strengths in the chlorophylls. Photochem Photobiol 77(5):497-501

König C, Neugebauer J (2012) Quantum chemical description of absorption properties and excited-state processes in photosynthtetic systems. ChemPhysChem 13:386-425

Krausz E, Hughes JL, Smith P, Pace R, Årsköld SP (2005) Oxygenevolving photosystem II core complexes: a new paradigm based on the spectral identification of the charge separating state, the primary acceptor and assignment of low-temperature fluorescence. Photochem Photobiol Sci 4:744-753

Kreisbeck C, Kramer T (2012) Long-lived electronic coherence in dissipative exciton dynamics of light-harvesting complexes. J Phys Chem Lett 3:2828-2833

Krueger BP, Scholes GD, Fleming GR (1998) Calculation of couplings and energy-transfer pathways between the pigments of LH2 by the ab initio transition density cube method. J Phys Chem B 102:5378-5386

Kühn O, Renger T, May V (1996) Theory of exciton-vibrational dynamics in molecular dimers. Chem Phys 204:99-114
Law CJ, Cogdell RJ (2008) The light-harvesting system of purple anoxygenic photosynthetic bacteria. In: Renger G (eds) Primary processes of photosynthesis. RSC Publishing, Cambridge, pp 205-259

Limantara L, Sakamoto Y, Koyama Y, Nagae H (1997) Effects of nonpolar and polar solvents on the $\mathrm{Q}_{x}$ and $\mathrm{Q}_{y}$ energies of bacteriochlorophyll $a$ and bacteriopheophytin $a$. Photochem Photobiol 65:330-337

Linke M, Lauer A, von Heimberger T, Zacarias A, Heyne K (2008) Three-dimensional orientation of the $\mathrm{Q}_{\mathrm{y}}$ transition dipole moment within the chlorophyll a molecule determined by femtosecond polarization resolved VIS pump-IR probe spectroscopy. J Am Chem Soc 130:14,904-14,905

Louwe RJW, Vrieze J, Hoff AJ, Aartsma TJ (1997) Toward an integral interpretation of the optical steady-state spectra of the FMO-complex of Prosthecochloris aestuarii. 2. Exciton simulations. J Phys Chem B 101:11280-11287

MacColl R (1998) Cyanobacterial phycobilisomes. J Struc Biol 124:311-334

Madjet ME, Abdurahman A, Renger T (2006) Intermolecular coulomb couplings from ab initio electrostatic potentials: application to optical transitions of strongly coupled pigments in photosynthetic antennae and reaction centers. J Phys Chem B 110:17,268-17,281

Madjet ME, Müh F, Renger T (2009) Deciphering the influence of short-range electronic couplings on optical properties of molecular dimers: application to special pairs in photosynthesis. J Phys Chem B 113:12,603-12,614

Müh F, Renger T (2012) Refined structure-based simulation of plant light-harvesting complex II: linear optical spectra of trimers and aggregates. Biochim Biophys Acta 1817:1446-1460

Müh F, Renger T (2013) Structure-based calculation of pigmentprotein and excitonic pigment-pigment coupling in photosynthetic light-harvesting complexes. In: van der Est A, Golber J (eds) The biophysics of photosynthesis. Springer (in press)

Müh F, Zouni A (2005) Extinction coefficients and critical solubilisation concentrations of photosystems I and II from Thermosynechococcus elongatus. Biochim Biophys Acta 1708:219-228

Müh F, Madjet ME, Adolphs J, Abdurahman A, Rabenstein B, Ishikita H, Knapp EW, Renger T (2007) $\alpha$-Helices direct excitation energy flow in the Fenna-Matthews-Olson protein. Proc Natl Acad Sci USA 104:16862-16867

Müh F, Madjet ME, Renger T (2010) Structure-based identification of energy sinks in plant light-harvesting complex II. J Phys Chem B 114:13517-13535

Müh F, Madjet ME, Renger T (2012) Structure-based simulation of linear optical spectra of the CP43 core antenna of photosystem II. Photosynth Res 111:87-101

Mukai K, Abe S, Sumi H (1999) Theory of rapid excitation energy transfer from B800 to optically forbidden exciton states of B850 in the antenna system $\mathrm{LH} 2$ of photosynthetic purple bacteria. J Phys Chem B 103:6096-6102

Nalbach P, Braun D, Thorwart M (2011) Exciton transfer dynamics and quantumness of energy transfer in the Fenna-MatthewsOlson complex. Phys Rev E 84:041926

Neugebauer J (2009) Subsystem-based theoretical spectroscopy of biomolecules and biomolecular assemblies. ChemPhysChem 10:3148-3173

Novoderezhkin V, Yakovlev AG, van Grondelle R, Shuvalov VA (2004) Coherent nuclear and electronic dynamics in primary charge separation in photosynthetic reaction centers: a Redfield theory approach. J Phys Chem B 108:7445-7457

Novoderezhkin VI, van Grondelle R (2010) Physical origins and models of energy transfer in photosynthetic light-harvesting. Phys Chem Chem Phys 12:7352-7365 
Novoderezhkin VI, Dekker JP, van Grondelle R (2007) Mixing of exciton and charge-transfer states in photosystem II reaction centers: modeling of stark spectra with modified redfield theory. Biophys J 93:1293-1311

Olbrich C, Strümpfer J, Schulten K, Kleinekathöfer U (2011) Quest for spatially correlated fluctuations in the FMO light-harvesting complex. J Phys Chem B 115:758-764

Olbrich C, Strümpfer J, Schulten K, Kleinekathöfer U (2011) Theory and simulation of the environmental effects on FMO electronic transitions. J Phys Chem Lett 2:1771-1776

Pawlowicz NP, Groot ML, van Stokkum IHM, van Grondelle R (2007) Charge separation and energy transfer in the photosystem II core complex studied by femtosecond midinfrared spectroscopy. Biophys J 93:2732-2742

Raszewski G, Renger T (2008) Light harvesting in photosystem II core complexes is limited by the transfer to the trap: Can the core complex turn into a photoprotective mode? J Am Chem Soc 130:4431-4446

Raszewski G, Saenger W, Renger T (2005) Theory of optical spectra of photosystem II reaction centers: location of the triplet state and the identity of the primary electron donor. J Phys Chem B 88:986-998

Redfield AG (1957) On the theory of relaxation processes. IBM Journal Res Dev 1:19-31

Renge I, Mauring K (2013) Spectral shift mechanism of chlorophylls in liquids and proteins. J Biol Chem 102:301-313

Renger G (2008) Overview of primary processes of photosynthesis. In: Renger G (eds) Primary processes of photosynthesis. RSC Publishing, Cambridge, pp 5-38

Renger T (2012) Photophysics of photosynthetic reaction centers. In: Wydrzynski TJ, Hillier W (eds) Molecular solar fuels. RSC Publishing, Cambridge, pp 143-159

Renger T, Marcus RA (2002) On the relation of protein dynamics and exciton relaxation in pigment-protein complexes: an estimation of the spectral density and a theory for the calculation of optical spectra. J Chem Phys 116:9997-10,019

Renger T, Marcus RA (2003) Variable-range hopping electron transfer through disordered bridge states: application to DNA. J Chem Phys 107:8404-8419

Renger T, May V (1998) Ultrafast exciton motion in photosynthetic antenna systems: the FMO-complex. J Phys Chem A 102:4381-4391

Renger T, Müh F (2012) Theory of excitonic couplings in dielectric media. Photosynth Res 111:47-52

Renger T, Müh F (2013) Understanding photosynthetic lightharvesting: a bottom up theoretical approach. Phys Chem Chem Phys 15:3348-3371

Renger T, Schlodder E (2010) Primary photophysical processes in photosystem II: bridging the gap between crystal structure and optical spectra. ChemPhysChem 11:1141-1153

Renger T, Grundkötter B, Madjet ME, Müh F (2008) Theory of solvatochromic shifts in nonpolar solvents reveals a new spectroscopic rule. Proc Natl Acad Sci USA 105(36):13,235-13,240

Renger T, Madjet ME, Müh F, Trostmann I, Schmitt FJ, Theiss C, Paulsen H, Eichler HJ, Knorr A, Renger G (2009) Thermally activated superradiance and intersystem crossing in the watersoluble chlorophyll binding protein. J Phys Chem B 113:9948-9957

Renger T, Klinger A, Steinecker F, Schmidt am Busch M, Numata J, Müh F (2012) Normal mode analysis of the spectral density of the Fenna-Matthews-Olson light-harvesting protein: how the protein dissipates the excess energy of excitons. J Phys Chem B 116:14565-14580

Rivera E, Montemayor D, Masia M, Coker D (2013) Influence of sitedependent pigment-protein interaction on excitation energy transfer in photosynthetic light-harvesting. J Phys Chem B 117:5510-5521

Roszak W, Howard TD, Southall J, Gardiner AT, Law CJ, Isaacs NW, Cogdell RJ (2003) Crystal structure of the RC-LH1 core complex from Rhodopseudomonas palustris. Science 302:1969-1972

Savhikin S, Buck D, Struve WS (1997) Oscillating anisotropies in a bacteriochlorophyll protein: evidence for quantum beating between exciton levels. Chem Phys 223:303-312

Schmidt am Busch M, Knapp EW (2005) One-electron reduction potential for oxygen- and sulfur-centered organic radicals in protic and aprotic solvents. J Am Chem Soc 127:15,730-15,737

Schmidt am Busch M, Müh F, Madjet ME, Renger T (2011) The eighth bacteriochlorophyll completes the excitation energy funnel in the FMO protein. J Phys Chem Lett 2:93-98

Scholes GD, Gould IR, Cogdell RJ, Fleming GR (1999) Ab initio molecular orbital calculations of electronic couplings in the LH2 bacterial light-harvesting complex of Rps. acidophila. J Phys Chem B 103:2543-2553

Senn HM, Thiel W (2007) QM/MM methods for biological systems. Top Curr Chem 268:173-290

Shelnutt JA, Song XZ, Ma JG, Jia SL, Jentzen W, Medforth CJ (1998) Nonplanar pophyrins and their significance in proteins. Chem Soc Rev 27:31-41

Shim S, Rebentrost P, Valleau S, Aspuru-Guzik AA (2012) Atomistic study of the long-lived quantum coherences in the FennaMatthews-Olson complex. Biophys J 102:649-660

Simonson T, Perahia D (1995) Microscopic dielectric properties of cytochrome c from molecular dynamics simulations in aqueous solution. J Am Chem Soc 117:7987-8000

Stein T, Kronik L, Baer R (2009) Reliable prediction of charge transfer excitations in molecular complexes using time-dependent density functional theory. J Am Chem Soc 131:2818-2820

Stowell M, McPhillips TM, Rees D, Soltis S, Abresch E, Feher G (1997) Light-induced structural changes in photosynthetic reaction center: implications for mechanism of electron-proton transfer. Science 276:812-816

Sundström V, van Grondelle R, Bergström H, Akesson E, Gillbro T (1986) Excitation energy transport in bacteriochlorophyll antenna systems of Rhodospirillum rubrum and Rhodobacter sphaeroides studied by low-intensity picosecond absorption spectroscopy. Biochim Biophys Acta 851:431-446

Sundström V, Pullerits T, van Grondelle R (1999) Photosynthetic light-harvesting: reconciling dynamics and structure of purple bacterial LH2 reveals function of photosynthetic unit. J Phys Chem B 103:2327-2346

Tronrud DE, Wen J, Gay L, Blankenship RE (2009) The structural basis for the difference in absorbance spectra for the FMO antenna protein from various green sulfur bacteria. Photosynth Res 100:79-87

Ullmann GM, Knapp EW (1999) Electrostatic models for computing protonation and redox equilibria in proteins. Eur Biophys $\mathbf{J}$ 28:533-550

Umena Y, Kawakami K, Shen J, Kamiya N (2011) Crystal structure of oxygen-evolving photosystem II at a resolution of $1.9 \AA$. Nature 473:55-60

van Grondelle R, Novoderezhkin VI (2006) Energy transfer in photosynthesis: experimental insights and quantitative models. Phys Chem Chem Phys 8:793-807

van Grondelle R, Dekker JP, Gillbro T, Sundström V (1994) Energy transfer and trapping in photosynthesis. Biochim Biophys Acta 1187:1-65

Visscher KJ, Bergström H, Sundström V, Hunter CN, van Grondelle $R$ (1989) Temperature dependence of energy transfer from the long wavelength antenna BChl-896 to the reaction center in Rhodospririllum rubrum, Rhodobacter sphaeroides (WT and 
M21 mutant) from $77 \mathrm{~K}$ to $177 \mathrm{~K}$, studied by picosecond absorption spectroscopy. Photosynth Res 22:211-217

Warshel A, Parson WW (1987) Spectroscopic properties of photosynthetic reaction centers. I. Theory. J Am Chem Soc 109:6143-6152

Wen J, Zhang H, Gross ML, Blankenship RE (2009) Membrane orientation of the FMO antenna protein from Chlorobaculum tepidum as determined by mass spectrometry-based footprinting. Proc Natl Acad Sci USA 106:6134-6139

Wendling M, Pullerits T, Przyjalgowski M, Vulto S, Aartsma T, van Grondelle R, van Amerongen H (2000) Electron-vibrational coupling in the Fenna-Matthews-Olson complex of Prosthecochloris aestuarii determined by temperature-dependent absorption and fluorescence line-narrowing measurements. J Phys Chem B 104:5825-5831
Wendling M, Przyjalgowski MA, Gülen D, Vulto SIE, Aartsma TJ, van Grondelle R, van Amerongen H (2002) The quantitative relationship between structure and polarized spectroscopy in the FMO complex of Prosthechochloris aestuarii: refining experiments and simulations. Photosynth Res 71:99-123

Yang M, Fleming GR (2002) Influence of phonons on exciton transfer dynamics: comparison of the Redfield, Förster, and modified Redfield equations. Chem Phys 275:355-372

Zhang WM, Meier T, Chernyak V, Mukamel S (1998) Excitonmigration and three-pulse femtosecond optical spectroscopies of photosynthetic antenna complexes. J Chem Phys 108:7763-7774

Zucchelli G, Santabarbara S, Jennings RC (2012) The $Q_{y}$ absorption spectrum of the light-harvesting complex II as determined by structure-based analysis of chlorophyll macrocycle deformations. Biochemistry 51:2717-2736 